TITLE:

\title{
Structure-activity relationship of imidazothiadiazole analogs for the binding to the ecdysone receptor of insect cells.
}

\section{$\operatorname{AUTHOR(S):~}$}

Yokoi, Taiyo; Minami, Saki; Nakagawa, Yoshiaki; Miyagawa, Hisashi

\section{CITATION:}

Yokoi, Taiyo ... [et al]. Structure-activity relationship of imidazothiadiazole analogs for the binding to the ecdysone receptor of insect cells.. Pesticide biochemistry and physiology 2015, 120: 40-50

\section{ISSUE DATE:}

2015-05

URL:

http://hdl.handle.net/2433/200903

\section{RIGHT:}

(c) 2014. This manuscript version is made available under the CC-BY-NC-ND 4 . license

http://creativecommons.org/licenses/by-nc-nd/4.0/; The full-text file will be made open to the public on 31 May 2016 in accordance with publisher's 'Terms and Conditions for Self-Archiving'; This is not the published version. Please cite only the published version.; この論文は出版社版でありません。引用の際には出版社版をご確認ご利用ください。 
1

2 Structure-activity relationship of imidazothiadiazole analogs for the

3 binding to the ecdysone receptor of insect cells

4

5 Taiyo Yokoi, Saki Minami, Yoshiaki Nakagawa*, and Hisashi Miyagawa

6

$7 \quad$ Division of Applied Life Sciences

8 Graduate School of Agriculture

$9 \quad$ Kyoto University

10 Kyoto 606-8502, Japan

11

$12 *$ Corresponding author

13 naka@kais.kyoto-u.ac.jp

14

15 


\section{Abstract}

17 Diacylhydrazines are the first non-steroidal ecdysone agonists, and five compounds are used as insecticides in agriculture. After the discovery of diacylhydrazine-type compounds, numerous non-steroidal structures were reported as ecdysone agonists.

20 Among various ecdysone agonists, imidazothiadiazoles are reported to be very potent in vitro; however the experimental detail for the structure identification and bioassays are not stated in the paper (Holmwood and Schindler, Bioorg. Med. Chem., 17, 4064-4070, 2009). In our present study, we synthesized 18 imidazothiadiazole-type compounds and confirmed the chemical structures by spectrometric analyses. The binding activity of the synthesized compounds to the ecdysone receptor was evaluated in terms of the concentration required for $50 \%$ inhibition of $\left[{ }^{3} \mathrm{H}\right]$ ponasterone $\mathrm{A}$ incorporation $\left[\mathrm{IC}_{50}(\mathrm{M})\right]$ into lepidopteran (Sf-9), coleopteran (BCRL-Lepd-SL1), and dipteran (NIAS-AeA12) 28 cells. 6-(2-Chlorophenyl)-2-(trifluoro methyl)imidazo[2,1-b]

29 [1,3,4]-thiadiazolyl-5-yl)acrylamide analogs with -CONHR (secondary amide) were

30 very potent against Sf-9 cells, but further alkylation (tertiary amide: $-\mathrm{CONR}_{2}$ ) decreased 31 the activity dramatically. Additionally, a primary amide analog $\left(-\mathrm{CONH}_{2}\right)$ was inactive.

32 The activity also decreased 150 -fold by the saturation of olefin region of the acrylamide 33 moiety. In addition, various substituents were introduced at the 2-position of the 34 imidazothiadiazole ring to disclose the physicochemical properties of the substituents which are important for receptor binding. The activity increased by 7500 -fold with the

36 introduction of the $\mathrm{CF}_{2} \mathrm{CF}_{2} \mathrm{CF}_{3}$ group compared to the unsubstituted compound against 37 Sf-9 cells. Quantitative structure-activity relationship analysis for these substituents 38 indicated that hydrophobic and electron-withdrawing groups were favorable for binding. 39 Some of the compounds with strong receptor binding activity showed good larvicidal 40 activity against Spodoptera litura. In contrast, the binding affinity of imidazothiadiazole analogs was low or not observed against dipteran and coleopteran cells. 


\section{Keywords:}

45 ecdysone agonists, molting inhibitor, imidazothiadiazole, Sf-9, ecdysone receptor 46 


\section{Introduction}

Arthropods, including insects, grow by repeated molting, which is regulated by molting hormones such as 20-hydroxyecdysone (20E; Fig. 1). Steroidal compounds with 20E-like activity are categorized as ecdysteroids, and have been identified in plants, animals, and microorganisms. To date, more than 400 ecdysteroids have been characterized (http://ecdybase.org), but no ecdysteroids have been launched as insecticides. Using steroids as insecticides may not be practical because of their high cost and synthesis difficulty. In addition, steroids do not easily penetrate the integument and are rapidly excreted from insects.

The discovery of diacylhydrazine (DAH)-type compounds (Fig. 1) enabled the development of novel ecdysone agonist insecticides [1,2]. Currently, five DAHs, namely, tebufenozide, methoxyfenozide, chromafenozide, fufenozide, and halofenozide, are available on the market. These DAH-type compounds are generally used in agriculture against Lepidoptera, but halofenozide also shows control of Coloeoptera.

Because the insecticidal spectrum of DAHs is narrow, other chemical structures have been screened as ecdysone agonists [3]. Among them, tetrahydroquinoline (THQ) [4], $N$-alkyl-3,5-di-tert-butyl-4-hydroxy-benzamide [5], $\alpha$-acylaminoketone [6], oxadiazoline [7], and $\gamma$-methylene- $\gamma$-lactam [8] have been described over the past two decades. In 2009, Holmwood and Schindler reported that imidazole (IMD) and imidazothiadiazole (ITD)-type compounds are ecdysone agonists (Fig. 2) [9]. Although the biological activity was evaluated quantitatively in terms of $\operatorname{pInd}_{50}(\mathrm{EcR}$ induction assay), experimental procedures and target insect species have not been described. Analytical data for the synthesized chemicals were not reported. 
75 however, thought to differ from those of DAHs and steroidal agonists such as ponasterone

A (PonA). The ITD substructure is very interesting, because some ITD-type compounds are reported to show anti-inflamatory [10], anticancer [11] and antitubercular activity [12].

The aim of this study was to quantitatively measure the ligand-receptor binding activity of ITD analogs and discuss the structure-activity relationship (SAR). For the SAR study, various ITD analogs were chemically synthesized. The substituents $X$ at 2-position of imidazothiadiazole ring (Fig. 2) were substituted with $\mathrm{H}, \mathrm{CH}_{3}, \mathrm{CF}_{3}, \mathrm{CF}_{2} \mathrm{CF}_{3}$, $\mathrm{CF}_{2} \mathrm{CF}_{2} \mathrm{CF}_{3}, \mathrm{SCH}_{3}, \mathrm{~S}(=\mathrm{O}) \mathrm{CH}_{3}, \mathrm{SO}_{2} \mathrm{CH}_{3}$, and the amide moiety to vary primary, secondary, and tertiary structure (Fig. 2). The linker between the imidazole ring and amide moiety was fixed as either trans $-\mathrm{CH}=\mathrm{CH}-$ or $-\mathrm{CH}_{2} \mathrm{CH}_{2^{-}}$(Fig. 2). Thioamide and sulfonamide analogs were also synthesized (Fig. 2). The binding affinity of these compounds was measured to the ecdysone receptors of three insect cells. The effect of substituents $\mathrm{X}$ on ligand-receptor binding against Sf-9 was quantitatively analyzed using classical quantitative structure-activity relationship (QSAR) analysis (Hansch-Fujita method) [13]. Docking simulation was also performed to predict the ligand-receptor interaction of ITDs.

Fig. 2

\section{Materials and Methods}

\subsection{Synthesis}

\subsubsection{Chemicals}

Chemicals were purchased from Sigma-Aldrich Co. (St. Louis, MO, USA), Tokyo Chemical Industry Co., Ltd. (Tokyo, Japan), Wako Pure Chemical Industries, Ltd. (Osaka, Japan), and Nacalai Tesque Inc. (Kyoto, Japan). Oven-dried glassware and positive argon pressure were used to maintain anhydrous conditions. Anhydrous solvents were 
103 commercially available and stored over molecular sieves. Flash column chromatography 104 was conducted using Wakogel ${ }^{\circledR}$ C-300HG (Wako Pure Chemical Industries, Osaka, 105 Japan) as the absorbent. NMR spectra were recorded on a Bruker AVANCE-400 or 106 Bruker AVANCE-500 spectrometer. Tetramethylsilane was used as the internal standard 107 for ${ }^{1} \mathrm{H}$ NMR (0 ppm); deuterated solvent signals were used as the internal standard for ${ }^{13} \mathrm{C}$ 108 NMR (77.16 ppm for $\mathrm{CDCl}_{3}$ and $39.52 \mathrm{ppm}$ for DMSO- $\left.d_{6}\right)$; and $\alpha, \alpha, \alpha$-trifluorotoluene 109 was used as the external standard for ${ }^{19} \mathrm{~F}$ NMR (-64.00 ppm). Melting points were 110 measured with a Yanaco melting point apparatus (Yanagimoto Seisakusho Co. Ltd., 111 Kyoto, Japan) and are uncorrected. Elemental analyses were performed at the 112 Microanalytical Center of Kyoto University. High-resolution mass spectra (HRMS) were 113 recorded on a Thermo Fisher Scientific EXACTIVE spectrometer at Department of 114 Synthetic Chemistry and Biological Chemistry of Kyoto University.

\subsubsection{Synthesis of 2 -amino-1,3,4-thiadiazoles (Scheme 1)}

i) 2-Amino-5-(trifluoromethyl)-1,3,4-thiadiazole (Step a): Phosphoryl chloride (27.5 mL, $121300 \mathrm{mmol}$ ) was added dropwise to a mixture of thiosemicarbazide (13.7 $\mathrm{g}, 150 \mathrm{mmol})$ and trifluoroacetic acid $(24.1 \mathrm{~mL}, 315 \mathrm{mmol})$ at $0^{\circ} \mathrm{C}$, and the mixture was gradually

123 heated to $70^{\circ} \mathrm{C}$. Foaming was observed during the reaction. The mixture was kept at $70^{\circ} \mathrm{C}$ 124 for 1 hour after the foaming ceased. After cooling the reaction mixture to room 125 temperature, it was treated with water $(300 \mathrm{~mL})$ and neutralized with saturated $\mathrm{Na}_{2} \mathrm{CO}_{3}$ 126 solution. The resulting solid was filtered off, washed with water and dried in vacuo to 127 yield an off-white solid (19.4 g, 77\%). ${ }^{1} \mathrm{H}$ NMR (400 MHz, DMSO- $\left.d_{6}\right) \delta 8.07$ (2H, s) 128 ppm; ${ }^{13} \mathrm{C}$ NMR $\left(100 \mathrm{MHz}, \mathrm{DMSO}-d_{6}\right) \delta 119.8\left(\mathrm{q}, J_{\mathrm{C}-\mathrm{F}}=269 \mathrm{~Hz}\right), 143.8\left(\mathrm{q}, J_{\mathrm{C}-\mathrm{F}}=37 \mathrm{~Hz}\right)$, 129171.7 ppm; ${ }^{19} \mathrm{~F}$ NMR (377 MHz, DMSO- $\left.d_{6}\right) \delta-59.77(3 \mathrm{~F}, \mathrm{~s}) \mathrm{ppm}$.

130 2-Amino-1,3,4-thiadiazoles with $\mathrm{CF}_{2} \mathrm{CF}_{3}$ and $\mathrm{CF}_{2} \mathrm{CF}_{2} \mathrm{CF}_{3}$ group were synthesized in a 
131 similar manner as above.

132

133 ii) 2-Amino-5-(methylthio)-1,3,4-thiadiazole (Step b): Potassium hydroxide (85\%, $3.4 \mathrm{~g}$,

$13451 \mathrm{mmol}$ ) was added in one portion to a suspension of 2-amino-5-mercapto-

135 1,3,4-thiadiazole $(6.7 \mathrm{~g}, 50 \mathrm{mmol})$ in 2-propanol $(10 \mathrm{~mL})$ and water $(7.5 \mathrm{~mL})$ at $0{ }^{\circ} \mathrm{C}$.

136 When the starting materials completely dissolved, methyl iodide (3.3 mL, $53 \mathrm{mmol})$ was

137 added dropwise to the reaction mixture maintaining the temperature below $15^{\circ} \mathrm{C}$. It was

138 stirred at room temperature overnight. The mixture was poured into water $(200 \mathrm{~mL})$ and

139 the resulting solid was filtered off. This was washed with water and dried in vacuo to 140 yield a white solid (5.6 g, 76\%). ${ }^{1} \mathrm{H}$ NMR (400 MHz, DMSO- $\left.d_{6}\right) \delta 2.58(3 \mathrm{H}, \mathrm{s}), 7.21(2 \mathrm{H}$,

$141 \mathrm{~s}) \mathrm{ppm} ;{ }^{13} \mathrm{C} \mathrm{NMR}\left(100 \mathrm{MHz}, \mathrm{DMSO}-d_{6}\right) \delta 16.6,151.9,168.9 \mathrm{ppm}$.

142

\section{$143 \quad$ 2.1.3. Synthesis of 2-chlorophenacyl bromide}

144 A solution of bromine $(26.5 \mathrm{~g}, 166 \mathrm{mmol})$ in acetic acid $(25 \mathrm{~mL})$ was added dropwise to a 145 solution of $2^{\prime}$-chloroacetophenone $(25.1 \mathrm{~g}, 162 \mathrm{mmol})$ in acetic acid $(175 \mathrm{~mL})$ at room 146 temperature. The mixture was stirred at room temperature for 2 hours. The mixture was 147 diluted with water $(250 \mathrm{~mL})$ and extracted with $\mathrm{CH}_{2} \mathrm{Cl}_{2}(250 \mathrm{~mL})$. The organic layer was 148 washed successively with water $(3 \times 250 \mathrm{~mL})$, saturated aqueous $\mathrm{NaHCO}_{3}$ solution $(250$ $149 \mathrm{~mL})$ and brine $(250 \mathrm{~mL})$, and dried over anhydrous $\mathrm{Na}_{2} \mathrm{SO}_{4}$. The solvent was evaporated 150 to give the crude 2-chlorophenacyl bromide (38.7 g, purity: ca. $83 \%$ determined by ${ }^{1} \mathrm{H}$ 151 NMR analysis), which was used for the next reaction without further purification.

153 2.1.4. Synthesis of (E)-3-(6-(2-chlorophenyl)-imidazo[2,1-b][1,3,4]thiadiazol-5-yl)154 acrylic acids (Scheme 2)

156 Scheme 2 
159 mixture of 2-amino-5-(trifluoromethyl)-1,3,4-thiadiazole (19.2 $\mathrm{g}, 114 \mathrm{mmol})$ and 160 2-chlorophenacyl bromide $(33.4 \mathrm{~g}, c a .120 \mathrm{mmol})$ in ethanol $(170 \mathrm{~mL})$ was refluxed 161 overnight. The mixture was then cooled in a freezer. The resulting crystalline solid was 162 filtered off, washed with cold ethanol, and dried in vacuo to yield a pale yellow solid 163 (19.3 g, 56\%). ${ }^{1} \mathrm{H}$ NMR (400 MHz, DMSO- $\left.d_{6}\right) \delta 7.39(1 \mathrm{H}, \mathrm{td}, J=7.9,1.8 \mathrm{~Hz}), 7.47(1 \mathrm{H}$, $164 \mathrm{td}, J=7.5,1.3 \mathrm{~Hz}), 7.58(1 \mathrm{H}, \mathrm{dd}, J=7.9,1.3 \mathrm{~Hz}), 8.11(1 \mathrm{H}, \mathrm{dd}, J=7.8,1.8 \mathrm{~Hz}), 8.96(1 \mathrm{H}$, 165 s) ppm.

166 Other imidazothiadiazole analogs with $\mathrm{CF}_{2} \mathrm{CF}_{3}, \mathrm{CF}_{2} \mathrm{CF}_{2} \mathrm{CF}_{3}, \mathrm{H}, \mathrm{CH}_{3}$, and $\mathrm{SCH}_{3}$ were 167 synthesized in a similar manner as above.

168

ii) 6-(2-Chlorophenyl)-2-(trifluoromethyl)imidazo[2,1-b][1,3,4]thiadiazole-5-carbaldehyde (Step b): Under an argon atmosphere, phosphoryl chloride (3.9 mL, $43 \mathrm{mmol}$ ) was added dropwise to anhydrous DMF $(20 \mathrm{~mL})$ at $0^{\circ} \mathrm{C}$ and stirred for $5 \mathrm{~min}$. To this was added 6-(2-chlorophenyl)-2-(trifluoromethyl)imidazo[2,1-b][1,3,4]thiadiazole (3.91 g,

$17313 \mathrm{mmol})$ in anhydrous DMF $(15 \mathrm{~mL})$, and the mixture was heated to $70^{\circ} \mathrm{C}$ and stirred overnight. It was poured into ice-water $(100 \mathrm{~mL})$, neutralized with saturated aqueous

$175 \mathrm{Na}_{2} \mathrm{CO}_{3}$ solution and then extracted with toluene $(1 \times 100 \mathrm{~mL}, 2 \times 50 \mathrm{~mL})$. The combined organic layer was washed with water $(3 \times 100 \mathrm{~mL})$ and brine $(100 \mathrm{~mL})$, and dried over

177 anhydrous $\mathrm{MgSO}_{4}$. The solvent was evaporated and the crude product was purified by 178 flash column chromatography (hexane/ethyl acetate $=95: 5-50: 50$ ) to yield a pale yellow 179 solid (2.91 g, 68\%). ${ }^{1} \mathrm{H}$ NMR (400 MHz, $\left.\mathrm{CDCl}_{3}\right) \delta 7.43-7.49(2 \mathrm{H}, \mathrm{m}), 7.55-7.59$ (2H, m), $1809.85(1 \mathrm{H}, \mathrm{s}) \mathrm{ppm}$.

181 Other imidazothiadiazole-5-carbaldehyde analogs with $\mathrm{CF}_{2} \mathrm{CF}_{3}, \mathrm{CF}_{2} \mathrm{CF}_{2} \mathrm{CF}_{3}, \mathrm{H}, \mathrm{CH}_{3}$, and $\mathrm{SCH}_{3}$ were synthesized in a similar manner as above.

iii) (E)-3-(6-(2-Chlorophenyl)-2-(trifluoromethyl)imidazo[2,1-b][1,3,4] thiadiazol-5-yl) acrylic acid (Step c): 6-(2-Chlorophenyl)-2-(trifluoromethyl)imidazo[2,1-b][1,3,4] 
187 dissolved in pyridine $(17 \mathrm{~mL})$. To this, malonic acid $(0.76 \mathrm{~g}, 7.3 \mathrm{mmol})$ was added and the 188 mixture was stirred at $100^{\circ} \mathrm{C}$ for $4 \mathrm{~h}$. After cooling, the reaction mixture was poured into

$1891 \mathrm{M} \mathrm{HCl}(70 \mathrm{~mL})$ and acidified with concentrated $\mathrm{HCl}$. The resulting solid was filtered 190 off, washed with water, and dried in vacuo. This solid was triturated in hexane/ether $(1: 1)$ 191 to give an off-white solid (1.89 g, 84\%). ${ }^{1} \mathrm{H}$ NMR (400 MHz, DMSO- $\left.d_{6}\right) \delta 6.68(1 \mathrm{H}, \mathrm{d}, J$ $192=16.0 \mathrm{~Hz}), 7.31(1 \mathrm{H}, \mathrm{d}, J=16.0 \mathrm{~Hz}), 7.52-7.61(3 \mathrm{H}, \mathrm{m}), 7.67-7.71(1 \mathrm{H}, \mathrm{m}), 12.64(1 \mathrm{H}, \mathrm{br}$. 193 s) ppm.

194 Other (imidazothiadiazol-5-yl)acrylic acid analogs with $\mathrm{CF}_{2} \mathrm{CF}_{3}, \mathrm{CF}_{2} \mathrm{CF}_{2} \mathrm{CF}_{3}, \mathrm{H}, \mathrm{CH}_{3}$, 195 and $\mathrm{SCH}_{3}$ were synthesized in a similar manner as above.

196

2.1.5. Synthesis of $(E)-3-(6-(2-c h l o r o p h e n y l) i m i d a z o[2,1-b][1,3,4]$ thiadiazol-5-yl) acrylamides (Scheme 3)

199

200

Scheme 3

201

202

203

i) (E)-3-(6-(2-Chlorophenyl)-2-(trifluoromethyl)imidazo[2,1-b][1,3,4]thiadiazol-5-yl)

204 acrylamide (1): Oxalyl chloride $(0.17 \mathrm{~mL}, 2.0 \mathrm{mmol})$ was added to the suspension of 205 (E)-3-(6-(2-chlorophenyl)-2-(trifluoromethyl)imidazo[2,1-b][1,3,4]thiadiazol-5-yl)acry206 lic acid (377 mg, $1.0 \mathrm{mmol})$ in $\mathrm{CH}_{2} \mathrm{Cl}_{2}(5 \mathrm{~mL})$ containing one drop of DMF. After the gas 207 evolution ceased, the mixture was refluxed for 2 hours. After cooling, the solvent was 208 evaporated to give the crude acid chloride. This was dissolved in $\mathrm{CH}_{2} \mathrm{Cl}_{2}(5 \mathrm{~mL})$, and then 209 added dropwise to vigorously stirred aqueous $\mathrm{NH}_{3}$ solution $(28 \%, 5 \mathrm{~mL})$ at $0^{\circ} \mathrm{C}$. The 210 mixture was stirred at room temperature overnight. It was diluted with water $(30 \mathrm{~mL})$ and 211 extracted with $\mathrm{CH}_{2} \mathrm{Cl}_{2}(3 \times 30 \mathrm{~mL})$. The combined organic layer was washed with water $212(50 \mathrm{~mL})$ and brine $(50 \mathrm{~mL})$, and dried over anhydrous $\mathrm{Na}_{2} \mathrm{SO}_{4}$. The solvent was 213 evaporated and the crude product was recrystallized from ethyl acetate/hexane to give a 214 white solid (276 mg, 73\%). Mp: $232-234^{\circ} \mathrm{C} .{ }^{1} \mathrm{H} \mathrm{NMR}\left(400 \mathrm{MHz}, \mathrm{CDCl}_{3}\right) \delta 5.67$ (2H, br 
$215 \mathrm{~s}), 6.96(1 \mathrm{H}, \mathrm{d}, J=15.6 \mathrm{~Hz}), 7.34-7.47(3 \mathrm{H}, \mathrm{m}), 7.51-7.58(2 \mathrm{H}, \mathrm{m}) \mathrm{ppm} ;{ }^{13} \mathrm{C} \mathrm{NMR}(100$ $\left.216 \mathrm{MHz}_{\mathrm{CDCl}}\right) \delta 118.7\left(\mathrm{q}, J_{\mathrm{C}-\mathrm{F}}=272 \mathrm{~Hz}\right), 119.0,122.9,126.2,127.2,130.5,130.9,131.5$, $217132.6,134.0,146.5,148.5,151.3$ (q, $\left.J_{\mathrm{C}-\mathrm{F}}=42 \mathrm{~Hz}\right), 167.3 \mathrm{ppm} ;{ }^{19} \mathrm{~F}$ NMR $(377 \mathrm{MHz}$, $\left.218 \mathrm{CDCl}_{3}\right) \delta-62.46(3 \mathrm{~F}, \mathrm{~s})$ ppm. Anal. Calcd for $\mathrm{C}_{14} \mathrm{H}_{8} \mathrm{ClF}_{3} \mathrm{~N}_{4} \mathrm{OS}: \mathrm{C}, 45.11 ; \mathrm{H}, 2.16$; , 219 15.03. Found: C, 45.07; H, 2.20; N, 15.05.

220

ii) (E)-3-(6-(2-Chlorophenyl)-2-(trifluoromethyl)imidazo[2,1-b][1,3,4]thiadiazol-5-yl)N-isopropylacrylamide (2): To a suspension of compound 12 (374 $\mathrm{mg}, 1.0 \mathrm{mmol})$ in anhydrous $\mathrm{CH}_{2} \mathrm{Cl}_{2}(5 \mathrm{~mL})$, EDC hydrochloride $(227 \mathrm{mg}, 1.2 \mathrm{mmol})$ and catalytic amount of DMAP were added. Then, isopropylamine $(98 \mu \mathrm{L}, 1.2 \mathrm{mmol})$ was added and the mixture was stirred at room temperature overnight. The mixture was diluted with $\mathrm{CH}_{2} \mathrm{Cl}_{2}$ $(15 \mathrm{~mL})$ and washed successively with saturated aqueous $\mathrm{Na}_{2} \mathrm{CO}_{3}$ solution, water, $1 \mathrm{M}$

$227 \mathrm{HCl}$, water, and brine $\left(10 \mathrm{~mL}\right.$ each). The organic layer was dried over $\mathrm{MgSO}_{4}$ and 228 concentrated to give compound 17 as pale yellow foam (387 mg, 93\%). This was further recrystallized from ethyl acetate/hexane to afford white crystals, which were used for the bioassays. Mp: $174-175^{\circ} \mathrm{C} .{ }^{1} \mathrm{H}$ NMR $\left(400 \mathrm{MHz}, \mathrm{CDCl}_{3}\right) \delta 1.22(6 \mathrm{H}, \mathrm{d}, J=6.6 \mathrm{~Hz})$, 4.14-4.27 (1H, m), $5.58(1 \mathrm{H}$, br d, $J=7.7 \mathrm{~Hz}), 6.86(1 \mathrm{H}, \mathrm{d}, J=15.5 \mathrm{~Hz}), 7.33-7.45(3 \mathrm{H}$, m), 7.50-7.54 (2H, m) ppm; ${ }^{13} \mathrm{C}$ NMR $\left(100 \mathrm{MHz}, \mathrm{CDCl}_{3}\right) \delta 22.9,41.9,118.7\left(\mathrm{q}, J_{\mathrm{C}-\mathrm{F}}=\right.$ $272 \mathrm{~Hz}), 120.6,123.2,124.6,127.1,130.5,130.7,131.6,132.6,134.1,146.1,148.0$, $151.0\left(\mathrm{q}, J_{\mathrm{C}-\mathrm{F}}=42 \mathrm{~Hz}\right), 164.6 \mathrm{ppm} ;{ }^{19} \mathrm{~F} \mathrm{NMR}\left(377 \mathrm{MHz}, \mathrm{CDCl}_{3}\right) \delta-62.43(3 \mathrm{~F}, \mathrm{~s}) \mathrm{ppm}$. Anal. Calcd for $\mathrm{C}_{17} \mathrm{H}_{14} \mathrm{ClF}_{3} \mathrm{~N}_{4} \mathrm{OS}$ : C, 49.22; H, 3.40; N, 13.51. Found: C, 49.16; H, 3.59; N, 13.65.

237 Other acrylamide analogs $(\mathbf{3}$ - 13) were synthesized in a similar manner to that described 238 for compound 2. Analytical data for the compounds are shown below.

240 iii) (E)-3-(6-(2-Chlorophenyl)-2-(trifluoromethyl)imidazo[2,1-b][1,3,4]thiadiazol-5-yl)241 N-cyclobutylacrylamide (3): Mp: 199-200 ${ }^{\circ} \mathrm{C} .{ }^{1} \mathrm{H}$ NMR (400 MHz, $\left.\mathrm{CDCl}_{3}\right) \delta 1.69-1.78$ $242(2 \mathrm{H}, \mathrm{m}), 1.88-2.00(2 \mathrm{H}, \mathrm{m}), 2.34-2.43(2 \mathrm{H}, \mathrm{m}), 4.51(1 \mathrm{H}$, quin, $J=8.1 \mathrm{~Hz}), 5.92(1 \mathrm{H}, \mathrm{br}$, 
$243 J=7.7 \mathrm{~Hz}), 6.86(1 \mathrm{H}, \mathrm{d}, J=15.5 \mathrm{~Hz}), 7.33-7.44(3 \mathrm{H}, \mathrm{m}), 7.50-7.54(2 \mathrm{H}, \mathrm{m}) \mathrm{ppm} ;{ }^{13} \mathrm{C}$ $244 \operatorname{NMR}\left(100 \mathrm{MHz}, \mathrm{CDCl}_{3}\right) \delta 15.3,31.4,45.2,118.7$ (q, $\left.J_{\mathrm{C}-\mathrm{F}}=272 \mathrm{~Hz}\right), 120.3,123.2,124.9$, $245127.1,130.5,130.8,131.6,132.6,134.1,146.2,148.1,151.0$ (q, $\left.J_{\mathrm{C}-\mathrm{F}}=42 \mathrm{~Hz}\right), 164.5$ 246 ppm; ${ }^{19} \mathrm{~F}$ NMR $\left(377 \mathrm{MHz}, \mathrm{CDCl}_{3}\right) \delta-62.43(3 \mathrm{~F}, \mathrm{~s})$ ppm. Anal. Calcd for $247 \quad \mathrm{C}_{18} \mathrm{H}_{14} \mathrm{ClF}_{3} \mathrm{~N}_{4} \mathrm{OS}: \mathrm{C}, 50.65 ; \mathrm{H}, 3.31 ; \mathrm{N}, 13.13$. Found: C, 50.52; H, 3.28; N, 13.25.

248

iv) (E)-3-(6-(2-Chlorophenyl)-2-(trifluoromethyl)imidazo[2,1-b][1,3,4] thiadiazol-5-yl) 250 -N-cyclohexylacrylamide (4): Mp: $221-223^{\circ} \mathrm{C} .{ }^{1} \mathrm{H} \mathrm{NMR}\left(400 \mathrm{MHz}, \mathrm{CDCl}_{3}\right) \delta 1.11-1.28$ $(3 \mathrm{H}, \mathrm{m}), 1.32-1.46(2 \mathrm{H}, \mathrm{m}), 1.59-1.69(1 \mathrm{H}, \mathrm{m}), 1.60-1.79(2 \mathrm{H}, \mathrm{m}), 1.94-2.00(2 \mathrm{H}, \mathrm{m})$, 252 3.83-3.94 (1H, m), $5.71(1 \mathrm{H}, \mathrm{br} \mathrm{d}, J=8.1 \mathrm{~Hz}), 6.88(1 \mathrm{H}, \mathrm{d}, J=15.6 \mathrm{~Hz}), 7.32-7.44(3 \mathrm{H}$, m), 7.49-7.55 (2H, m) ppm; ${ }^{13} \mathrm{C} \mathrm{NMR}\left(100 \mathrm{MHz}, \mathrm{CDCl}_{3}\right) \delta 25.0,25.7,33.3,48.8,118.7$

254 $\left(\mathrm{q}, J_{\mathrm{C}-\mathrm{F}}=272 \mathrm{~Hz}\right), 120.8,123.2,124.5,127.0,130.5,130.7,131.6,132.6,134.0,146.1$, 255 148.0, $151.0\left(\mathrm{q}, J_{\mathrm{C}-\mathrm{F}}=42 \mathrm{~Hz}\right) \mathrm{ppm} ; 164.5,164.5 .{ }^{19} \mathrm{~F}$ NMR $\left(377 \mathrm{MHz}, \mathrm{CDCl}_{3}\right) \delta-62.42$ 256 (3F, s) ppm. Anal. Calcd for $\mathrm{C}_{20} \mathrm{H}_{18} \mathrm{ClF}_{3} \mathrm{~N}_{4} \mathrm{OS}: \mathrm{C}, 52.81 ; \mathrm{H}, 3.99 ; \mathrm{N}, 12.32$. Found: $\mathrm{C}$, 257 $52.82 ; \mathrm{H}, 4.02 ; \mathrm{N}, 12.32$.

258

259

v) (E)-3-(6-(2-Chlorophenyl)-2-(trifluoromethyl)imidazo[2,1-b][1,3,4]thiadiazol-5-yl)260 N-phenylacrylamide (5): $\mathrm{Mp:} 222-224^{\circ} \mathrm{C} .{ }^{1} \mathrm{H} \mathrm{NMR}\left(400 \mathrm{MHz}, \mathrm{CDCl}_{3}\right) \delta 7.03-7.15(2 \mathrm{H}$, 261 m), 7.29-7.47 (5H, m), 7.50-7.53 (1H, m), 7.57-7.69 (4H, m) ppm; ${ }^{13} \mathrm{C}$ NMR (100 MHz, 262 $\left.\mathrm{CDCl}_{3}\right) \delta 118.7\left(\mathrm{q}, J_{\mathrm{C}-\mathrm{F}}=272 \mathrm{~Hz}\right), 119.9,120.3,123.1,124.6,126.0,127.1,129.2,130.5$, 263 $130.9,131.4,132.5,134.0,138.1,146.5,148.6,151.3\left(\mathrm{q}, J_{\mathrm{C}-\mathrm{F}}=42 \mathrm{~Hz}\right), 163.7 \mathrm{ppm} ;{ }^{19} \mathrm{~F}$ 264 NMR (377 MHz, $\left.\mathrm{CDCl}_{3}\right) \delta-62.37$ (3F, s) ppm. Anal. Calcd for $\mathrm{C}_{20} \mathrm{H}_{12} \mathrm{ClF}_{3} \mathrm{~N}_{4} \mathrm{OS}: \mathrm{C}$, 265 53.52; H, 2.69; N, 12.48. Found: C, 53.25; H, 2.88; N, 12.43.

266

267

vi) (E)-3-(6-(2-Chlorophenyl)-2-(trifluoromethyl)imidazo[2,1-b][1,3,4] thiadiazol-5-yl) 268 -N-isopropyl-N-methylacrylamide (6): Mp: $144-145^{\circ} \mathrm{C} .{ }^{1} \mathrm{H} \mathrm{NMR}\left(400 \mathrm{MHz}, \mathrm{CDCl}_{3}\right) \delta$ 269 (mixture of rotamers) $1.14(3.3 \mathrm{H}, \mathrm{d}, J=6.6 \mathrm{~Hz}), 1.26(2.7 \mathrm{H}, \mathrm{d}, J=6.6 \mathrm{~Hz}), 2.89(1.35 \mathrm{H}, \mathrm{s})$, 270 $2.98(1.65 \mathrm{H}, \mathrm{s}), 4.27(0.45 \mathrm{H}$, sep, $J=6.6 \mathrm{~Hz}), 4.96(0.55 \mathrm{H}$, sep, $J=6.6 \mathrm{~Hz}), 7.32-7.53$ 
$271(6 \mathrm{H}, \mathrm{m}) \mathrm{ppm} ;{ }^{13} \mathrm{C} \mathrm{NMR}\left(100 \mathrm{MHz}, \mathrm{CDCl}_{3}\right) \delta$ (mixture of rotamers) 19.5, 20.7, 26.6, 28.5, $27244.6,28.5,118.3,118.5,118.7$ (q, $\left.J_{\mathrm{C}-\mathrm{F}}=272 \mathrm{~Hz}\right), 122.8,123.7,125.4,125.7,127.1,130.4$, $273130.7,131.7,132.6,134.1,145.9,146.0,147.3,147.6,150.9\left(\mathrm{q}, J_{\mathrm{C}-\mathrm{F}}=42 \mathrm{~Hz}\right), 165.8$, $274166.1 \mathrm{ppm} ;{ }^{19} \mathrm{~F}$ NMR $\left(377 \mathrm{MHz}, \mathrm{CDCl}_{3}\right) \delta$ (mixture of rotamers) -62.81 (1.35F, s), -62.71 275 (1.65F, s) ppm. Anal. Calcd for $\mathrm{C}_{18} \mathrm{H}_{16} \mathrm{ClF}_{3} \mathrm{~N}_{4} \mathrm{OS}$ : C, 50.41; H, 3.76; N, 13.06. Found: C, $276 \quad 50.43 ; \mathrm{H}, 3.76 ; \mathrm{N}, 12.94$.

277

278

vii) (E)-3-(6-(2-Chlorophenyl)-2-(trifluoromethyl)imidazo[2,1-b][1,3,4] thiadiazol-5-yl) 279 -1-(piperidin-1-yl)prop-2-en-1-one (7): Mp: 168-170 ${ }^{\circ} \mathrm{C} .{ }^{1} \mathrm{H} \mathrm{NMR}\left(400 \mathrm{MHz}, \mathrm{CDCl}_{3}\right) \delta$ 280 1.55-1.75 (6H, m), 3.50-3.70 (4H, m), 7.33-7.46 (4H, m), 7.51-7.54 (2H, m) ppm; ${ }^{13} \mathrm{C}$ 281 NMR $\left(100 \mathrm{MHz}, \mathrm{CDCl}_{3}\right) \delta 24.8,25.7,26.9,43.6,47.2,117.6,118.7$ (q, $\left.J_{\mathrm{C}-\mathrm{F}}=271 \mathrm{~Hz}\right)$, 282 $123.7,125.8,127.1,130.4,130.7,131.7,132.6,134.1,145.9,147.5,150.9$ (q, $J_{\mathrm{C}-\mathrm{F}}=42$ 283 $\mathrm{Hz}$ ), 165.0 ppm; ${ }^{19} \mathrm{~F}$ NMR (377 MHz, $\left.\mathrm{CDCl}_{3}\right) \delta-62.67$ (3F, s) ppm. Anal. Calcd for 284 $\mathrm{C}_{19} \mathrm{H}_{16} \mathrm{ClF}_{3} \mathrm{~N}_{4} \mathrm{OS}: \mathrm{C}, 51.76 ; \mathrm{H}, 3.66 ; \mathrm{N}, 12.71$. Found: C, 51.86; H, 3.81; N, 12.71.

285

286

viii) (E)-3-(6-(2-Chlorophenyl)-2-(trifluoromethyl)imidazo[2,1-b][1,3,4] thiadiazol-5-yl) 287 -1-morpholinoprop-2-en-1-one (8): Mp: $171-172{ }^{\circ} \mathrm{C} .{ }^{1} \mathrm{H} \mathrm{NMR}\left(400 \mathrm{MHz}, \mathrm{CDCl}_{3}\right) \delta$ 288 3.60-3.70 (8H, m), 7.35-7.46 (4H, m), 7.52-7.54 (1H, m), $7.60(1 \mathrm{H}, \mathrm{d}, J=16.0 \mathrm{~Hz}) \mathrm{ppm}$; 289 ${ }^{13} \mathrm{C} \mathrm{NMR}\left(100 \mathrm{MHz}, \mathrm{CDCl}_{3}\right) \delta 42.7,46.3,67.0,116.1,118.7$ (q, $\left.J_{\mathrm{C}-\mathrm{F}}=272 \mathrm{~Hz}\right), 123.5$, 290 $126.7,127.1,130.5,130.8,131.6,132.6,134.0,146.3,148.1,151.2$ (q, $J_{\mathrm{C}-\mathrm{F}}=42 \mathrm{~Hz}$ ), 291 165.2 ppm. ${ }^{19} \mathrm{~F}$ NMR (377 MHz, $\left.\mathrm{CDCl}_{3}\right) \delta-62.61$ (3F, s) ppm. Anal. Calcd for 292 $\mathrm{C}_{18} \mathrm{H}_{14} \mathrm{ClF}_{3} \mathrm{~N}_{4} \mathrm{O}_{2} \mathrm{~S}: \mathrm{C}, 48.82 ; \mathrm{H}, 3.19 ; \mathrm{N}, 12.65$. Found: C, 48.67; H, 3.27; N, 12.55.

293

294

ix) (E)-3-(6-(2-Chlorophenyl)-2-(perfluoroethyl)imidazo[2,1-b][1,3,4]thiadiazol-5-yl)$295 \quad$-isopropylacrylamide (9): Mp: $165-166^{\circ} \mathrm{C} .{ }^{1} \mathrm{H} \mathrm{NMR}\left(400 \mathrm{MHz}, \mathrm{CDCl}_{3}\right) \delta 1.22(6 \mathrm{H}, \mathrm{d}, J$ $296=6.6 \mathrm{~Hz}), 4.12-4.27(1 \mathrm{H}, \mathrm{m}), 5.58(1 \mathrm{H}, \mathrm{br} \mathrm{d}, J=7.8 \mathrm{~Hz}), 6.80(1 \mathrm{H}, \mathrm{d}, J=15.5 \mathrm{~Hz})$, 297 7.33-7.48 (3H, m), 7.50-7.58 (2H, m) ppm; ${ }^{13} \mathrm{C}$ NMR $\left(100 \mathrm{MHz}, \mathrm{CDCl}_{3}\right) \delta 22.9,41.9$, 298 $109.3(\mathrm{tq}, J=256,41 \mathrm{~Hz}), 118.0\left(\mathrm{qt}, J_{\mathrm{C}-\mathrm{F}}=285,36 \mathrm{~Hz}\right), 120.6,123.1,124.6,127.1,130.5$, 
$299130.8,131.5,132.6,134.0,146.2,148.0,150.7\left(\mathrm{t}, J_{\mathrm{C}-\mathrm{F}}=31 \mathrm{~Hz}\right), 164.6 \mathrm{ppm} ;{ }^{19} \mathrm{~F}$ NMR $300\left(377 \mathrm{MHz}, \mathrm{CDCl}_{3}\right) \delta-111.16\left(2 \mathrm{~F}, \mathrm{q}, J_{\mathrm{F}-\mathrm{F}}=2.3 \mathrm{~Hz}\right),-84.20\left(3 \mathrm{~F}, \mathrm{t}, J_{\mathrm{F}-\mathrm{F}}=2.3 \mathrm{~Hz}\right) \mathrm{ppm}$. Anal. 301 Calcd for $\mathrm{C}_{18} \mathrm{H}_{14} \mathrm{ClF}_{5} \mathrm{~N}_{4} \mathrm{OS}$ : C, 46.51; H, 3.04; N, 12.05. Found: C, 46.50; H, 3.08; N, $302 \quad 12.26$.

303

304 x) (E)-3-(6-(2-Chlorophenyl)-2-(perfluoropropyl)imidazo[2,1-b][1,3,4]thiadiazol-5-yl)$305 \quad$-isopropylacrylamide (10): $\mathrm{Mp}: 170-172^{\circ} \mathrm{C} .{ }^{1} \mathrm{H} \mathrm{NMR}\left(500 \mathrm{MHz}, \mathrm{CDCl}_{3}\right) \delta 1.21(6 \mathrm{H}, \mathrm{d}$, $306 J=6.6 \mathrm{~Hz}), 4.15-4.26(1 \mathrm{H}, \mathrm{m}), 5.67(1 \mathrm{H}, \mathrm{br} \mathrm{d}, J=7.8 \mathrm{~Hz}), 6.82(1 \mathrm{H}, \mathrm{d}, J=15.5 \mathrm{~Hz})$, 307 7.33-7.45 (3H, m), 7.50-7.58 (2H, m) ppm; ${ }^{13} \mathrm{C}$ NMR (125 MHz, $\left.\mathrm{CDCl}_{3}\right) \delta 22.9,41.9$, $308 \quad 105.5-111.2(\mathrm{~m}), 111.3\left(\mathrm{tt}, J_{\mathrm{C}-\mathrm{F}}=285,36 \mathrm{~Hz}\right), 117.6\left(\mathrm{qt}, J_{\mathrm{C}-\mathrm{F}}=286,34 \mathrm{~Hz}\right), 120.7,123.2$, $309124.6,127.1,130.5,130.7,131.6,132.6,134.1,146.2,148.0,150.6\left(\mathrm{t}, J_{\mathrm{C}-\mathrm{F}}=31 \mathrm{~Hz}\right)$, $310164.6 \mathrm{ppm}$; due to ${ }^{13} \mathrm{C}-{ }^{19} \mathrm{~F}$ coupling, the signals of the perfluoropropyl group were so 311 weak that it was difficult to detect those signals and their coupling patterns. ${ }^{19} \mathrm{~F}$ NMR $312\left(471 \mathrm{MHz}, \mathrm{CDCl}_{3}\right) \delta-126.61\left(2 \mathrm{~F}, \mathrm{t}, J_{\mathrm{F}-\mathrm{F}}=8.5 \mathrm{~Hz}\right),-108.75\left(2 \mathrm{~F}, \mathrm{sex}, J_{\mathrm{F}-\mathrm{F}}=9.4 \mathrm{~Hz}\right),-81.01$ 313 (3F, t, $\left.J_{\mathrm{F}-\mathrm{F}}=9.6 \mathrm{~Hz}\right)$ ppm. Anal. Calcd for $\mathrm{C}_{19} \mathrm{H}_{14} \mathrm{ClF}_{7} \mathrm{~N}_{4} \mathrm{OS}: \mathrm{C}, 44.33 ; \mathrm{H}, 2.74 ; \mathrm{N}, 10.88$. 314 Found: C, 44.18; H, 2.79; N, 10.89.

315

316 xi) (E)-3-(6-(2-Chlorophenyl)imidazo[2,1-b][1,3,4]thiadiazol-5-yl)-N-isopropylacryl317 amide (11): Mp: $210-212^{\circ} \mathrm{C} .{ }^{1} \mathrm{H} \mathrm{NMR}\left(400 \mathrm{MHz}, \mathrm{CDCl}_{3}\right) \delta 1.19(6 \mathrm{H}, \mathrm{d}, J=6.6 \mathrm{~Hz})$, $3184.12-4.26(1 \mathrm{H}, \mathrm{m}), 5.65(1 \mathrm{H}, \mathrm{br} \mathrm{d}, J=7.6 \mathrm{~Hz}), 6.92(1 \mathrm{H}, \mathrm{d}, J=15.5 \mathrm{~Hz}) 7.30-7.39(2 \mathrm{H}$, $319 \mathrm{~m}), 7.42-7.47(1 \mathrm{H}, \mathrm{m}), 7.48-7.57(2 \mathrm{H}, \mathrm{m}), 8.69(1 \mathrm{H}, \mathrm{s}) \mathrm{ppm} ;{ }^{13} \mathrm{C} \mathrm{NMR}(100 \mathrm{MHz}$, $\left.320 \mathrm{CDCl}_{3}\right) \delta 22.9,41.7,119.5,122.6,125.2,126.9,130.30,130.32,132.2,132.6,134.0$, 321 146.2, 147.3, 147.5, 165.1 ppm. HRMS (ESI) m/z: $\mathrm{C}_{16} \mathrm{H}_{16} \mathrm{ClN}_{4} \mathrm{OS}[\mathrm{M}+\mathrm{H}]^{+}$, calcd 322347.0728 , found 347.0717 .

323

324 xii) (E)-3-(6-(2-Chlorophenyl)-2-methylimidazo[2,1-b][1,3,4]thiadiazol-5-yl)-N325 isopropylacrylamide (12): Mp: $214-215^{\circ} \mathrm{C} .{ }^{1} \mathrm{H}$ NMR (400 MHz, $\left.\mathrm{CDCl}_{3}\right) \delta 1.20(6 \mathrm{H}, \mathrm{d}, J$ $326=6.5 \mathrm{~Hz}), 2.78(3 \mathrm{H}, \mathrm{s}), 4.20(1 \mathrm{H}, \mathrm{m}), 5.59(1 \mathrm{H}$, br d, $J=7.7 \mathrm{~Hz}), 6.89(1 \mathrm{H}, \mathrm{d}, J=15.4 \mathrm{~Hz})$, 
327 7.28-7.38 (2H, m), 7.40-7.54 (3H, m) ppm; ${ }^{13} \mathrm{C}$ NMR (100 MHz, $\left.\mathrm{CDCl}_{3}\right) \delta 18.0,23.0$, $32841.7,119.1,122.3,125.5,126.9,130.1,130.3,123.4,132.7,134.1,146.2,147.6,160.2$, 329165.3 ppm. Anal. Calcd for $\mathrm{C}_{17} \mathrm{H}_{17} \mathrm{ClN}_{4} \mathrm{OS}: \mathrm{C}, 56.58 ; \mathrm{H}, 4.75 ; \mathrm{N}, 15.53$. Found: $\mathrm{C}, 56.73$; $330 \quad \mathrm{H}, 4.65 ; \mathrm{N}, 15.70$.

331

332 xiii) (E)-3-(6-(2-Chlorophenyl)-2-(methylthio)imidazo[2,1-b][1,3,4]thiadiazol-5-yl)333 N-isopropylacrylamide (13): $\mathrm{Mp}: 229-230^{\circ} \mathrm{C} .{ }^{1} \mathrm{H} \mathrm{NMR}\left(400 \mathrm{MHz}, \mathrm{CDCl}_{3}\right) \delta 1.19(6 \mathrm{H}$, $334 \mathrm{~d}, J=6.6 \mathrm{~Hz}), 2.79(3 \mathrm{H}, \mathrm{s}), 4.12-4.27(1 \mathrm{H}, \mathrm{m}), 5.62(1 \mathrm{H}, \mathrm{br} \mathrm{d}, J=7.7 \mathrm{~Hz}), 6.80(1 \mathrm{H}, \mathrm{d}, J$ $335=15.4 \mathrm{~Hz}), 7.29-7.38(2 \mathrm{H}, \mathrm{m}), 7.39-7.55(3 \mathrm{H}, \mathrm{m}) \mathrm{ppm} ;{ }^{13} \mathrm{C} \mathrm{NMR}\left(100 \mathrm{MHz}, \mathrm{CDCl}_{3}\right) \delta$ $33616.7,22.9,41.7,119.3,122.4,125.4,126.9,130.2,130.3,132.3,132.6,134.0,145.7$, 337 146.8, 162.6, 165.2 ppm. HRMS (ESI) $m / z: \mathrm{C}_{17} \mathrm{H}_{18} \mathrm{ClN}_{4} \mathrm{OS}_{2}[\mathrm{M}+\mathrm{H}]^{+}$, calcd 393.0605, 338 found 393.0594.

339

340

341

2.1.6. Synthesis of imidazothiadiazole analogs with $\mathrm{S}(=\mathrm{O}) \mathrm{CH}_{3}$ and $\mathrm{SO}_{2} \mathrm{CH}_{3}(\mathrm{Scheme}$ 4)

342

Sheme 4

343

344

i) (E)-3-(6-(2-Chlorophenyl)-2-(methylsulfinyl)imidazo[2,1-b][1,3,4]thiadiazol-5-yl)-

$345 \quad$ N-isopropylacrylamide (14):_Compound 13 (394 mg, $1.0 \mathrm{mmol}$ ) was dissolved in $\mathrm{CH}_{2} \mathrm{Cl}_{2}$

$346(5 \mathrm{~mL})$ and cooled to $0^{\circ} \mathrm{C}$. To this was added the solution of $m$-chloroperbenzoic acid

$347(70 \%, 246 \mathrm{mg}, 1.0 \mathrm{mmol})$ in $\mathrm{CH}_{2} \mathrm{Cl}_{2}(5 \mathrm{~mL})$ within $30 \mathrm{~min}$ and the mixture was stirred at 348 room temperature overnight. The reaction was quenched by adding saturated aqueous $349 \mathrm{Na}_{2} \mathrm{CO}_{3}$ solution $(5 \mathrm{~mL})$. The organic layer was washed with saturated aqueous $\mathrm{Na}_{2} \mathrm{CO}_{3}$ 350 solution $(5 \mathrm{~mL})$ and brine $(5 \mathrm{~mL})$, and dried over anhydrous $\mathrm{Na}_{2} \mathrm{SO}_{4}$. The solvent was 351 evaporated and the crude product was purified by flash column chromatography (ethyl 352 acetate $100 \%$ ) to yield white foam (348 mg, 85\%). This was further recrystallized from $353 \mathrm{CHCl}_{3} /$ hexane to afford white crystals, which were used for the bioassays. Mp: $354236-237^{\circ} \mathrm{C} .{ }^{1} \mathrm{H}$ NMR $\left(400 \mathrm{MHz}, \mathrm{CDCl}_{3}\right) \delta 1.20(6 \mathrm{H}, \mathrm{d}, J=6.6 \mathrm{~Hz}), 3.17(3 \mathrm{H}, \mathrm{s})$, 
355

356

357

358

359

360

361

362

363

364

365

366

367

368

369

370

371

372

373

374

375

376

377

378

379

380

381

382

4.12-4.28 (1H, m), $5.65(1 \mathrm{H}$, br d, $J=7.8 \mathrm{~Hz}), 6.77(1 \mathrm{H}, \mathrm{d}, J=15.7 \mathrm{~Hz}), 7.31-7.47(3 \mathrm{H}$, m), 7.47-7.57 (2H, m) ppm; ${ }^{13} \mathrm{C} \mathrm{NMR}\left(100 \mathrm{MHz}, \mathrm{CDCl}_{3}\right) \delta 22.9,41.8,43.9,120.1,122.6$, 124.8, 127.1, 130.4, 130.6, 131.8, 132.6, 134.0, 146.8, 147.1, 164.8, 173.5 ppm. HRMS (ESI) $m / z: \mathrm{C}_{17} \mathrm{H}_{18} \mathrm{ClN}_{4} \mathrm{O}_{2} \mathrm{~S}_{2}[\mathrm{M}+\mathrm{H}]^{+}$, calcd 409.0554, found 409.0546.

ii) (E)-3-(6-(2-Chlorophenyl)-2-(methylsulfonyl)imidazo[2,1-b][1,3,4]thiadiazol-5-yl)N-isopropylacrylamide (15): Compound 13 (398 mg, $1.0 \mathrm{mmol}$ ) was dissolved in $\mathrm{THF} / \mathrm{MeOH} /$ water $(1: 1: 1,15 \mathrm{~mL})$ and cooled to $0^{\circ} \mathrm{C}$. Oxone ${ }^{\circledR}(1.84 \mathrm{~g}, 3.0 \mathrm{mmol})$ was slowly added to the solution and the mixture was stirred at $0^{\circ} \mathrm{C}$ for $5 \mathrm{~min}$ and at room temperature overnight. After largely evaporating the solvent, the mixture was diluted with water $(20 \mathrm{~mL})$ and extracted with $\mathrm{CH}_{2} \mathrm{Cl}_{2}(1 \times 10 \mathrm{~mL}, 2 \times 5 \mathrm{~mL})$. The combined organic layer was washed with water $(10 \mathrm{~mL})$ and brine $(10 \mathrm{~mL})$, and dried over anhydrous $\mathrm{Na}_{2} \mathrm{SO}_{4}$. The solvent was evaporated and the crude product was purified by flash column chromatography (hexane/ethyl acetate $=3: 7$ ) to yield a pale yellow solid (340 mg, 79\%). This was further recrystallized from $\mathrm{CHCl}_{3} /$ hexane to afford pale yellow crystals, which were used for the bioassays. Mp: $247-249^{\circ} \mathrm{C} .{ }^{1} \mathrm{H}$ NMR (400 MHz, $\mathrm{CDCl}_{3}$ ) $\delta 1.20(6 \mathrm{H}, \mathrm{d}, J=6.6 \mathrm{~Hz}), 3.45(3 \mathrm{H}, \mathrm{s}), 4.12-4.27(1 \mathrm{H}, \mathrm{m}), 5.81(1 \mathrm{H}, \mathrm{br} \mathrm{d}, J=7.6 \mathrm{~Hz})$, $6.86(1 \mathrm{H}, \mathrm{d}, J=15.6 \mathrm{~Hz}), 7.33-7.47(3 \mathrm{H}, \mathrm{m}), 7.48-7.59(2 \mathrm{H}, \mathrm{m}) \mathrm{ppm} ;{ }^{13} \mathrm{C} \mathrm{NMR}(100 \mathrm{MHz}$, $\left.\mathrm{CDCl}_{3}\right) \delta 22.9,41.9,44.0,120.9,123.1,124.4,127.1,130.5,130.8,131.4,132.6,134.0$, 147.3, 148.2, 162.1, 164.6 ppm. HRMS (ESI) $m / z: \mathrm{C}_{17} \mathrm{H}_{18} \mathrm{ClN}_{4} \mathrm{O}_{3} \mathrm{~S}_{2}[\mathrm{M}+\mathrm{H}]^{+}$, calcd 425.0503, found 425.0492 .

\subsubsection{Synthesis of other imidazothiadiazole analogs}

Scheme 5

i) 3-(6-(2-Chlorophenyl)-2-(trifluoromethyl)imidazo[2,1-b][1,3,4]thiadiazol-5-yl)-Nisopropylpropanamide (16): To the solution of (E)-3-(6-(2-chlorophenyl)-2- 
383 (trifluoromethyl)imidazo[2,1-b][1,3,4]thiadiazol-5-yl)acrylic acid (374 mg, $1.0 \mathrm{mmol})$ in $384 \mathrm{MeOH}(6 \mathrm{~mL})$ was added hydrazine hydrate $(2.0 \mathrm{~mL}, 41 \mathrm{mmol})$ and catalytic amount of 385 acetic acid and saturated aqueous $\mathrm{CuSO}_{4}$ solution. To this, the solution of $\mathrm{NaIO}_{4}(1.09 \mathrm{~g}$, 386 $5.1 \mathrm{mmol})$ in water $(10 \mathrm{~mL})$ was added dropwise in 1 hour and the mixture was stirred at 387 room temperature overnight. After largely evaporating the solvent, the mixture was 388 diluted with $3 \mathrm{M} \mathrm{HCl}(20 \mathrm{~mL})$ and extracted with ethyl acetate $(3 \times 20 \mathrm{~mL})$. The combined organic layer was washed with water $(20 \mathrm{~mL})$ and brine $(20 \mathrm{~mL})$, and dried over anhydrous $\mathrm{Na}_{2} \mathrm{SO}_{4}$. The solvent was evaporated to give the crude propanoic acid. This was suspended in anhydrous $\mathrm{CH}_{2} \mathrm{Cl}_{2}(5 \mathrm{~mL})$, and EDC hydrochloride (225 mg, $1.2 \mathrm{mmol}$ ) 392 and catalytic amount of DMAP were added. Then, isopropylamine (98 $\mu \mathrm{L}, 1.2 \mathrm{mmol})$ was added and the mixture was stirred at room temperature overnight. Because TLC analysis indicated that the reaction was not complete, EDC hydrochloride (122 mg, 0.64 mmol), isopropylamine $(50 \mu \mathrm{L}, 0.61 \mathrm{mmol})$ and catalytic amount of DMAP were further added and the mixture was stirred at room temperature for 3 days. The mixture was diluted with $\mathrm{CH}_{2} \mathrm{Cl}_{2}(15 \mathrm{~mL})$ and washed successively with saturated aqueous $\mathrm{Na}_{2} \mathrm{CO}_{3}$ solution, water, $1 \mathrm{M} \mathrm{HCl}$, water, and brine $(10 \mathrm{~mL}$ each). The organic layer was dried over $\mathrm{MgSO}_{4}$ and filtered through a plug of silica gel, which was eluted with ethyl acetate. The filtrate was concentrated and the crude product was purified by flash column chromatography (hexane/ethyl acetate $=3: 2$ ) to yield a pale yellow solid ( $86 \mathrm{mg}, 21 \%$ ). This was further recrystallized from ethyl acetate to afford pale yellow crystals, which were used for the bioassays. Mp: $180-182^{\circ} \mathrm{C} .{ }^{1} \mathrm{H}$ NMR $\left(400 \mathrm{MHz}, \mathrm{CDCl}_{3}\right) \delta 1.09(6 \mathrm{H}, \mathrm{d}$, $J=6.6 \mathrm{~Hz}), 2.49-2.58(2 \mathrm{H}, \mathrm{m}), 3.22-3.31(2 \mathrm{H}, \mathrm{m}), 3.93-4.09(1 \mathrm{H}, \mathrm{m}), 5.16(1 \mathrm{H}, \mathrm{br} \mathrm{d}, J=$ $5.4 \mathrm{~Hz}), 7.32-7.40(2 \mathrm{H}, \mathrm{m}), 7.42-7.53(2 \mathrm{H}, \mathrm{m}) \mathrm{ppm} ;{ }^{13} \mathrm{C} \mathrm{NMR}\left(100 \mathrm{MHz}, \mathrm{CDCl}_{3}\right) \delta 20.6$, 406 22.8, 34.0, 41.6, 118.8 (q, $\left.J_{\mathrm{C}-\mathrm{F}}=271 \mathrm{~Hz}\right), 125.0,127.1,130.10,130.12,132.5,132.8$, C, 48.76; H, 4.01; N, 13.26 . 
Scheme 6

412

413 ii) E)-3-(6-(2-Chlorophenyl)-2-(trifluoromethyl)imidazo[2,1-b][1,3,4]thiadiazol-5-yl)-

$414 \quad$-isopropylprop-2-enethioamide (17): A mixture of compound 2 (349 mg, $0.84 \mathrm{mmol}$ ) 415 and Lawesson's reagent $(174 \mathrm{mg}, 0.43 \mathrm{mmol})$ in toluene $(20 \mathrm{~mL})$ was refluxed for 2 hours. 416 After cooling, the mixture was filtered through a plug of silica gel, which was eluted with 417 ethyl acetate. The filtrate was concentrated and the crude product was purified by flash 418 column chromatography (hexane/ethyl acetate $=3: 1$ ) and recrystallization from ethyl 419 acetate/hexane to yield yellow crystals (282 mg, 78\%). Mp: $203-204^{\circ} \mathrm{C} .{ }^{1} \mathrm{H}$ NMR (400 $\left.420 \mathrm{MHz}, \mathrm{CDCl}_{3}\right) \delta 1.33(6 \mathrm{H}, \mathrm{d}, J=6.6 \mathrm{~Hz}), 4.77-4.91(1 \mathrm{H}, \mathrm{m}), 7.16-7.26(2 \mathrm{H}, \mathrm{m}), 7.36-7.49$ $421(3 \mathrm{H}, \mathrm{m}), 7.52-7.58(1 \mathrm{H}, \mathrm{m}), 7.87(1 \mathrm{H}, \mathrm{d}, J=15.1 \mathrm{~Hz}) \mathrm{ppm} .{ }^{13} \mathrm{C} \mathrm{NMR}\left(100 \mathrm{MHz}, \mathrm{CDCl}_{3}\right)$ $422 \delta 21.7,47.6,118.7$ (q, $\left.J_{\mathrm{C}-\mathrm{F}}=272 \mathrm{~Hz}\right), 123.4,126.6,126.8,127.1,130.6,130.8,131.5$, $423132.6,133.9,146.2,148.7,151.1$ (q, $\left.J_{\mathrm{C}-\mathrm{F}}=42 \mathrm{~Hz}\right), 192.7 \mathrm{ppm} ;{ }^{19} \mathrm{~F}$ NMR $(377 \mathrm{MHz}$, $\left.424 \mathrm{CDCl}_{3}\right) \delta-62.35(3 \mathrm{~F}, \mathrm{~s})$ ppm. Anal. Calcd for $\mathrm{C}_{17} \mathrm{H}_{14} \mathrm{ClF}_{3} \mathrm{~N}_{4} \mathrm{~S}_{2}: \mathrm{C}, 47.39 ; \mathrm{H}, 3.28 ; \mathrm{N}$, 425 13.00. Found: C, 47.27; H, 3.29; N, 12.86.

426

Scheme 7

428

iii) (E)-2-(6-(2-Chlorophenyl)-2-(trifluoromethyl)imidazo $[2,1-b][1,3,4]$ thiadiazol-5yl)-N-isopropylethenesulfonamide (18): To a suspension of lithium chloride (319 mg, 7.5 mmol) and $\mathrm{Ph}_{2} \mathrm{P}(\mathrm{O}) \mathrm{CH}_{2} \mathrm{SO}_{2} \mathrm{NHBoc}(1.31 \mathrm{~g}, 3.3 \mathrm{mmol})$ in anhydrous $\mathrm{CH}_{3} \mathrm{CN}(25 \mathrm{~mL})$ was added 1,8-diazabicyclo[5.4.0]undec-7-ene (DBU; $1.1 \mathrm{~mL}, 7.4 \mathrm{mmol})$. To this

433 solution was added 6-(2-chlorophenyl)-2-(trifluoromethyl)imidazo[2,1-b][1,3,4]434 thiadiazole-5-carbaldehyde $(994 \mathrm{mg}, 3.0 \mathrm{mmol})$ and the mixture was stirred at room 435 temperature for $1.5 \mathrm{~h}$. The resulting suspension was diluted with water $(75 \mathrm{~mL})$ and the $436 \mathrm{pH}$ was adjusted to 3 with $1 \mathrm{M} \mathrm{HCl}$. This was extracted with ether $(1 \times 80 \mathrm{~mL}, 2 \times 40 \mathrm{~mL})$, 437 and the combined organic layer was washed with brine $(100 \mathrm{~mL})$ and dried over 438 anhydrous $\mathrm{MgSO}_{4}$. The solvent was evaporated and the crude product was purified by 
439 flash column chromatography (hexane/ethyl acetate $=95: 5-50: 50$ ) to give the $N$-Boc 440 vinyl sulfonamide as a pale yellow solid (1.11 g, 73\%). ${ }^{1} \mathrm{H}$ NMR (400 MHz, DMSO- $\left.d_{6}\right) \delta$ $4411.37(9 \mathrm{H}, \mathrm{s}), 7.24(1 \mathrm{H}, \mathrm{d}, J=15.4 \mathrm{~Hz}), 7.36(1 \mathrm{H}, \mathrm{d}, J=15.4 \mathrm{~Hz}), 7.52-7.62(3 \mathrm{H}, \mathrm{m})$, $442 \quad 7.68-7.72(1 \mathrm{H}, \mathrm{m}), 11.46(1 \mathrm{H}, \mathrm{br} \mathrm{s}) \mathrm{ppm}$.

$443 \quad N$-Boc vinyl sulfonamide obtained as above $(511 \mathrm{mg}, 1.0 \mathrm{mmol})$, isopropanol 444 (0.19 mL, $2.5 \mathrm{mmol})$ and triphenylphosphine (668 $\mathrm{mg}, 2.6 \mathrm{mmol})$ were dissolved in 445 anhydrous THF (15 mL). Diethyl azodicarboxylate (DEAD; 40\% in toluene; $1.35 \mathrm{~mL}$, $4463.1 \mathrm{mmol}$ ) was slowly added to the solution and the mixture was stirred at room 447 temperature for $1 \mathrm{~h}$. The solvent was evaporated and the crude product was purified by 448 flash column chromatography (hexane/ethyl acetate $=95: 5-65: 35$ ) to give the $N$-Boc $449 \quad \mathrm{~N}$-isopropyl vinyl sulfonamide as a pale yellow solid (416 mg, 76\%). ${ }^{1} \mathrm{H}$ NMR (400 MHz, $\left.450 \mathrm{CDCl}_{3}\right) \delta 1.41(6 \mathrm{H}, \mathrm{d}, J=6.9 \mathrm{~Hz}), 1.54(9 \mathrm{H}, \mathrm{s}), 4.55(1 \mathrm{H}, \mathrm{sep}, J=6.9 \mathrm{~Hz}), 7.38-7.48(4 \mathrm{H}$, $451 \mathrm{~m}), 7.53-7.58(1 \mathrm{H}, \mathrm{m}), 7.69(1 \mathrm{H}, \mathrm{d}, J=15.5 \mathrm{~Hz}) \mathrm{ppm}$. To a solution of $N$-Boc $N$-isopropyl vinyl sulfonamide obtained as above (377 mg, $4530.68 \mathrm{mmol})$ in $\mathrm{CH}_{2} \mathrm{Cl}_{2}(5 \mathrm{~mL})$ was added trifluoroacetic acid (TFA; $5 \mathrm{~mL}$ ) and the 454 mixture was stirred at room temperature for $1 \mathrm{~h}$. The solvent was evaporated and the 455 crude product was purified by flash column chromatography (hexane/ethyl acetate $=95: 5$ $456-50: 50)$ to give a white solid $(270 \mathrm{mg}, 88 \%)$. This was further recrystallized from $457 \mathrm{CHCl}_{3} /$ hexane to afford white crystals, which were used for the bioassays. Mp: 458 $157-158^{\circ} \mathrm{C} .{ }^{1} \mathrm{H}$ NMR $\left(500 \mathrm{MHz}, \mathrm{CDCl}_{3}\right) \delta 1.21(6 \mathrm{H}, \mathrm{d}, J=6.6 \mathrm{~Hz}), 3.51-3.62(1 \mathrm{H}, \mathrm{m})$, $4.42(1 \mathrm{H}, \mathrm{d}, J=7.7 \mathrm{~Hz}), 7.30(1 \mathrm{H}, \mathrm{d}, J=15.4 \mathrm{~Hz}), 7.35(1 \mathrm{H}, \mathrm{d}, J=15.4 \mathrm{~Hz}), 7.38-7.48$ 460 $(3 \mathrm{H}, \mathrm{m}), 7.52-7.56(1 \mathrm{H}, \mathrm{m}) \mathrm{ppm} ;{ }^{13} \mathrm{C} \mathrm{NMR}\left(125 \mathrm{MHz}, \mathrm{CDCl}_{3}\right) \delta 24.2,46.4,118.6\left(\mathrm{q}, J_{\mathrm{C}-\mathrm{F}}\right.$ 461 $=271 \mathrm{~Hz}), 120.9,124.4,126.2,127.3,130.6,131.1,131.2,132.5,133.8,147.1,148.9$, 462 $151.9\left(\mathrm{q}, J_{\mathrm{C}-\mathrm{F}}=42 \mathrm{~Hz}\right) \mathrm{ppm} ;{ }^{19} \mathrm{~F}$ NMR $\left(471 \mathrm{MHz}, \mathrm{CDCl}_{3}\right) \delta-62.52(3 \mathrm{~F}, \mathrm{~s}) \mathrm{ppm}$. Anal.

463 Calcd for $\mathrm{C}_{17} \mathrm{H}_{14} \mathrm{ClF}_{3} \mathrm{~N}_{4} \mathrm{~S}_{2}: \mathrm{C}, 47.39 ; \mathrm{H}, 3.28 ; \mathrm{N}, 13.00$. Found: $\mathrm{C}, 47.27 ; \mathrm{H}, 3.29 ; \mathrm{N}$, 464 12.86. HRMS (ESI) $m / z: \mathrm{C}_{16} \mathrm{H}_{15} \mathrm{ClF}_{3} \mathrm{~N}_{4} \mathrm{O}_{2} \mathrm{~S}_{2}[\mathrm{M}+\mathrm{H}]^{+}$, calcd 451.0272, found 451.0261 . 465 
467 Tritiated ponasterone A $\left(\left[{ }^{3} \mathrm{H}\right]\right.$ PonA, $\left.140 \mathrm{Ci} / \mathrm{mmol}\right)$ was purchased from American 468 Radiolabeled Chemicals Inc. (St. Louis, MO, USA). PonA and 20E were purchased 469 from Cosmo Bio Co., Ltd. (Tokyo, Japan) and Sigma-Aldrich Co. (St. Louis, MO, USA), 470 respectively. RH-5849 and tebufenozide were from our stock samples. All insect cells are 471 cultured in our laboratory. Originally, Sf-9 cell line was kindly gifted from Wakenyaku 472 Co., Ltd. (Kyoto, Japan), NIAS-AeA12 was given from NIAS Genebank (Tsukuba, 473 Japan)[14], and BCIRL-Lepd-SL1 was kindly gifted from Dr. Cynthia Goodman 474 (USDA-ARS, Columbia, MO, USA)[15].

475 The ligand binding assay using insect cells was performed as previously reported [16, 476 17]. In brief, insect cell suspension $\left(400 \mu \mathrm{L} ; 2-3 \times 10^{6}\right.$ cells $\left./ \mathrm{mL}\right)$ was incubated with 477 DMSO solution of a test compound $(1 \mu \mathrm{L})$ and $70 \%$ EtOH solution of $\left[{ }^{3} \mathrm{H}\right] \mathrm{PonA}(2 \mu \mathrm{L}$; 478 ca. $60,000 \mathrm{dpm})$ at $25^{\circ} \mathrm{C}$ for $30 \mathrm{~min}$. The mixture was diluted with water $(3 \mathrm{~mL})$ and filtered through a glass filter GF-75 (ADVANTEC, Tokyo, Japan). The filter was washed 2 times with water $(3 \mathrm{~mL})$, dried, and placed in a vial containing $3 \mathrm{~mL}$ of Insta-Gel Plus (PerkinElmer, Inc., Waltham, MA, USA) to measure the radioactivity with a LSC-6100 liquid scintillation counter (Aloka, Tokyo, Japan). The concentration required for $50 \%$ inhibiton of $\left[{ }^{3} \mathrm{H}\right]$ PonA binding $\left(\mathrm{IC}_{50}\right)$ was determined by probit analysis[18], and its reciprocal logarithm, $\mathrm{pIC}_{50}$, was used as the index of binding activity.

\subsection{Larvicidal activity test}

Spodoptera litura was kindly provided from Ishihara Sangyo Kaisha, Ltd. (Kusatsu, Japan). Twenty $3^{\text {rd }}$ instar larvae were put in the glass petri dish with paper filter. DMSO solution of a test compound $(1 \mu \mathrm{L})$ was applied on the dorsal part of larvae. They were reared in an insectary at $25^{\circ} \mathrm{C}$. Insecta LFS (Nosan Corporation Life-Tech Department, Yokohama, Japan) was used to feed larvae. After one week rearing, the mortality was measured, and 50\% lethal dose $\left(\mathrm{LD}_{50}\right)$ was determined by probit analysis[18]. Its reciprocal logarithm, $\mathrm{pLD}_{50}$, was used as the index of larvicidal activity. 
495

496

497

498

499

500

501

502

503

504

505

506

507

508

509

510

511

512

513

514

515

516

517

518

519

520

521

522

\subsection{QSAR analysis}

In order to examine the effect of the substituents at 2-position of the imidazothiadiazole ring, the Hansch-Fujita QSAR analysis was performed using QREG 2.05[19]. ClogP values of synthesized chemicals, which were shown in Table 1, were calculated by ClogP for Windows Ver. 4.0 (Biobyte Corp., Claremont, CA, USA). In all equations, the number in parentheses are $95 \%$ confidence intervals of each coefficient, $n$ is the number of compounds used to analyze, $s$ is the standard deviation, and $r$ is the correlation coefficient, and $F$ is the value of ratio between regression and residual variances.

\subsection{Receptor modeling and ligand-receptor docking}

Since no 3-D structure of Spodoptera frugiperda EcR (SfEcR) is available, we constructed a 3-D structure model of the ligand binding domain (LBD) of SfEcR from the X-ray structure of Lepidopteran EcR using a homology modeling software PDFAMS (In-Silico Sciences Inc., Tokyo, Japan)[20]. We combined two partial primary sequences of SfEcR (NCBI accession number: AAM54494 and CAD58232)[21, 22] to construct the primary sequence of SfEcR-LBD. At present four X-ray structures of Lepidopteran EcR bound to different ligands, namely, PonA (PDB ID: 1R1K) [23], 20E (2R40) [24], a DAH-type agonist BYI06830 (1R20)[23] and an imidazole-type agonist BYI08346 (3IXP), are available. We used 1R1K, 1R20 and 3IXP as the templates for homology modeling because the shapes of the ligand binding pockets (LBP) are different among them. Thus, we obtained three homology models of SfEcR, which were stored as complexes with PonA, BYI06830 or BYI08346 in order to compare the binding modes. Ligand-receptor docking was conducted using OMEGA (ver. 2.5.1.4)[25] and OEDocking (ver. 3.0.1) of Openeye Co. Ltd. (Santa Fe, NM, USA; http://www.eyesopen.com). First, the LBPs of the SfEcR homology models were defined using "MAKE RECEPTOR" tool of OEDocking. Next, the mol2 file of ITD (10) was processed with OMEGA to generate the conformer libraries. Generated conformers are 
523 aligned in the order of ascending energy and low energy conformers (maximum: 200)

524 were compiled into a single file as a conformer library. Finally, these conformers were 525 docked to the LBPs of SfEcR using "FRED"[26] tool of OEDocking. FRED uses

526 Chemgauss 4 scoring function, and this function ranks each binding mode in terms of 527 shape interactions, hydrogen bonding interactions, metal-chelator interactions, and 528 desolvation. The binding modes which gave the highest Chemgauss 4 scores towards each 529 LBPs of SfEcR were shown in Fig. 4.

\section{$531 \quad$ 3. Results}

\section{$532 \quad 3.1$ Synthesis}

533 ITDs were synthesized according to a previously described method with some 534 modifications [27]. 2-Amino-1,3,4-thiadiazoles bearing $\mathrm{CF}_{3}, \mathrm{CF}_{2} \mathrm{CF}_{3}$, and $\mathrm{CF}_{2} \mathrm{CF}_{2} \mathrm{CF}_{3}$ 535 groups were prepared using a reported method [28]. 536 2-Amino-5-(methylthio)-1,3,4-thiadiazole was also prepared according to a modified 537 method described in the literature [29]. Formation of the imidazo[2,1- $b][1,3,4]$ thiadiazole 538 ring was accomplished by simply refluxing 2-amino-1,3,4-thiadiazoles and 539 2-chlorophenacyl bromide in ethanol. While compounds with electron-withdrawing 540 substituents $\left(\mathrm{CF}_{3}, \mathrm{CF}_{2} \mathrm{CF}_{3}\right.$, and $\left.\mathrm{CF}_{2} \mathrm{CF}_{2} \mathrm{CF}_{3}\right)$ did not form $\mathrm{HBr}$ salts, compounds with 541 other substituents $\left(\mathrm{H}, \mathrm{CH}_{3}, \mathrm{SCH}_{3}\right)$ precipitated as $\mathrm{HBr}$ salts in the reaction mixture. In the 542 next step, imidazothiadiazoles were subjected to the Vilsmeier-Haack reaction to afford 543 imidazo[2,1-b][1,3,4]thiadiazole-5-carbaldehydes. Next, Knoevenagel condensation of 544 aldehydes with malonic acid afforded pure $(E)$-acrylic acids. Finally, condensation of 545 acrylic acids with various amines yielded ITDs (1-13). In further, compound 13 was oxidized with $m$-CPBA and Oxone ${ }^{\circledR}$ to yield sulfoxide 14 and sulfone 15.

547 Next, we modified the acrylamide moiety. Hydrogenation of the double bond in 548 the acrylic acid followed by condensation with isopropylamine afforded the 549 propionamide 16. Hydrogenation using Pd-C or Wilkinson's catalyst under a hydrogen 550 atmosphere was unsuccessful, but diimide reduction afforded the desired compound, 
551 although in poor yield. Compound $\mathbf{2}$ was successfully converted to thioamide $\mathbf{1 7}$ using 552 Lawesson's reagent. Vinyl sulfonamide 18 was then synthesized in three steps. First, the 553 intermediate aldehyde was subjected to the modified Horner-Wittig reaction [30] to

554 afford the $N$-Boc vinyl sulfonamide with complete $E$ selectivity. Use of LiCl and DBU 555 [31] rather than strong bases was essential for preventing product degradation. Next, $556 N$-Boc vinyl sulfonamide was alkylated at the acidic NH group through the Mitsunobu 557 reaction, and then Boc protecting group was removed by TFA to yield vinyl sulfonamide 55818.

559

\subsection{Ligand binding activity}

The biological activities of various ITD analogs are summarized in Table 1 . In terms of the inhibition of $\left[{ }^{3} \mathrm{H}\right]$ PonA incorporation to Sf-9 cells, compound $\mathbf{1}$ with the

563 primary acrylamide moiety at the 5-position of the imidazothiadiazole ring was inactive.

564 However, secondary amides with $i-\operatorname{Pr}(2), c-\mathrm{Bu}(3)$, and $c$-Hex (4) were very potent. $\mathrm{IC}_{50}$ 565 values of these compounds were approximately 10-20 nM, which was 10-20 times more 566 potent than the natural molting hormone $20 \mathrm{E}\left(\mathrm{IC}_{50}=200 \mathrm{nM}\right)$. The $\mathrm{Ph}$ analog $(5)$ was less 567 potent than the alkyl analogs (2-4) among secondary amides, but equipotent to 20E (19). 568 Further alkylation (tertiary acrylamide: 6-8) drastically decreased the activity. 569 Conversion of the oxygen atom of the amide moiety to a sulfur atom (thioamide: 17) did not have a large impact on activity, but saturation of the olefin moiety (16) decreased

571 binding by 100 -fold. Introduction of a sulfonamide moiety (18) drastically decreased 572 activity by approximately 1000 -fold compared to compound 2 .

Next, the substituent effect at the 2-position of the imidazothiadiazole ring was 577 examined for $\mathrm{CF}_{2} \mathrm{CF}_{3}(\mathbf{9}), \mathrm{CF}_{2} \mathrm{CF}_{2} \mathrm{CF}_{3}(\mathbf{1 0}), \mathrm{H}(\mathbf{1 1}), \mathrm{CH}_{3}(\mathbf{1 2}), \mathrm{SCH}_{3}(\mathbf{1 3}), \mathrm{S}(=\mathrm{O}) \mathrm{CH}_{3}(\mathbf{1 4})$, and $\mathrm{SO}_{2} \mathrm{CH}_{3}$ (15). By introducing strong electron withdrawing fluorinated alkyl groups 
579 such as $\mathrm{CF}_{3}, \mathrm{CF}_{2} \mathrm{CF}_{3}$, and $\mathrm{CF}_{2} \mathrm{CF}_{2} \mathrm{CF}_{3}$, the activity dramatically increased (more than

580 1000-fold compared to unsubstituted compound 11). Other electron withdrawing groups

581 containing a sulfur atom such as $\mathrm{S}(=\mathrm{O}) \mathrm{CH}_{3}$ and $\mathrm{SO}_{2} \mathrm{CH}_{3}$ did not enhance the activity. The

$582 \mathrm{SCH}_{3}$ group enhanced the activity by 300 -fold, although its electronic properties were

583 equivalent to $\mathrm{H}$ in terms of Hammett $\sigma$. The electron-donating $\mathrm{CH}_{3}$ group increased the

584 activity by only 5-fold. Among TDI congeners, compound $\mathbf{1 0}$ showed the highest activity,

585 which was 3-fold higher than that of PonA and only 3-fold less potent than tebufenozide 586 in the binding assay against Sf-9 cells. The structure-activity relationship is summarized 587 in Fig. 3.

Fig. 3

590

591

592

The binding assay was also performed using other insect cell lines, including

593 Colorado potato beetle cells (BCIRL-Lepd-SL) and Asian tiger mosquito cells 594 (NIAS-AeA12). As shown in Table 1, a few compounds showed moderate activity against 595 mosquito cells, but most compounds were weak or inactive against these cells. 596 Compound 9 was 2.5-fold more potent than tebufenozide against NIAS-AeA12, while it 597 was approximately 30 -fold less potent than PonA.

598

\subsection{Larvicidal activity}

600 Larvicidal activity of the synthesized compounds was measured against S. litura, 601 which is shown in Table 1 . The $\mathrm{pLD}_{50}$ values of compounds $\mathbf{2}$ and $\mathbf{9}$ were determined to 602 be 5.16 and 5.03, respectively, which were approximately $1 / 20$ of tebufenozide, but 603 5-fold more toxic than RH-5849.

604

605

\subsection{QSAR analysis}

As shown in Table 1, activity was enhanced by introducing substituents at the 
607 2-position of the imidazothiadiazole ring. To determine the physicochemical mechanism 608 of these substituents, binding activity was quantitatively analyzed using substituent 609 parameters. We previously demonstrated that the hydrophobicity of substituent is 610 important for the binding of DAHs to the ecdysone receptor of Sf-9 cells [32]. Therefore, 611 activity was quantitatively analyzed using hydrophobicity $\Delta \mathrm{C} \log \mathrm{P}[\mathrm{C} \log \mathrm{P}(\mathrm{X})-\mathrm{C} \log \mathrm{P}$ $612(\mathrm{H})]$ to formulate statistically significant values using Eq. 1.

613

614

$$
\begin{aligned}
\mathrm{pIC}_{50}=1.326( \pm 0.772) \Delta \mathrm{Clog} \mathrm{P}+6.162( \pm 0.828) \\
\mathrm{n}=8 \quad \mathrm{~s}=0.930 \quad \mathrm{r}=0.864 \quad \mathrm{~F}_{1,6}=17.688
\end{aligned}
$$

616

617 This equation suggests that the hydrophobic interaction between the substituents and the 618 ligand binding site of the receptor is important for binding. Although the equation was 619 significant according to the $F$ test and the $\Delta C \log \mathrm{P}$ term was justified over $99 \%$ by t-test, 620 this correlation equation was not acceptable because of the large standard deviation. 621 Because the $\mathrm{pIC}_{50}$ value was highly reproducible [32], the value of 0.930 is too large. 622 Therefore, the addition of another physicochemical parameter to Eq. 1 was considered, 623 although using two parameters may not be allowed for the analysis of eight compounds (n

$624=8$ ). Addition of an electronic parameter (Swain-Lupton F: field effect) drastically 625 improved the correlation, as shown in Eq. 2, although the standard deviation remained 626 large $(\mathrm{s}=0.349)$.

627

$$
\begin{gathered}
\mathrm{pIC}_{50}=1.519( \pm 0.315) \Delta \mathrm{Clog} \mathrm{P}+3.923( \pm 1.643) \mathrm{F}+4.872( \pm 0.631) \\
\mathrm{n}=8 \quad \mathrm{~s}=0.349 \quad \mathrm{r}=0.985 \quad \mathrm{~F}_{2,5}=81.754
\end{gathered}
$$

630

631 These results indicate that the electrostatic interaction between substituents and the 632 receptor surface surrounding the substituents is important for activity because the 633 correlation derived using $\mathrm{F}$ was better than that using Hammett $\sigma$. Physicochemical 634 parameter values and calculated $\mathrm{pIC}_{50}$ values from Eq. 2 are listed in Table 2. 
Table 2.

637

638

\section{Discussion}

We previously synthesized various non-steroidal ecdysone agonists, including

640 diacylhydrazine (DAH) [33-35], N-alkyl-3,5-di-tert-butyl-4-hydroxybenzamides

641 (DTBHIB) [36], tetrahydroquinoline (THQ)[14], oxadiazoline (ODZ) [3], and

$642 \gamma$-methylene- $\gamma$-lactam (GML) (Akahane unpublished), and measured their biological

643 activity against whole insects, insect tissue, insect cells, and in vitro translated EcR/USP

644 proteins. Some DAH analogs (tebufenozide, methoxyfenozide, and chromafenozide)

645 were very potent against lepidopteran tissues and proteins, were equipotent to

646 ponasterone A, and were moderately potent against dipteran tissues and proteins, but not

647 potent against Ceoleopteran. In contrast, THQ-type compounds were reported to be 648 potent against Diptera, particularly mosquitoes [4], but they were not very potent against

649 Lepidoptera [37]. As shown in Table 1, the selectivity of ITD-type compound 9 was

650 similar to that of the DAH-type compound 21 (tebufenozide). These results indicate that

651 the binding mode of ITDs may be similar to that of DAHs, but different from those of

652 THQs and steroidal agonists.

653 According to Holmwood and Schindler [9], the binding mode of ITDs differs

654 from those of PonA and DAHs, but there is no data supporting the binding mode of ITDs.

655 In contrast, the X-ray crystal structure of the EcR/USP complex bound to an IMD-type 656 compound can be found in the Protein Data Bank (PDB ID: 3IXP). We performed

657 docking simulation of ITD compounds to predict the ligand-receptor binding modes, and

658 two crystal structures of receptor complexes bound to PonA and a DAH-type compound 659 (BYI06833) were used as template 3-D structures (Fig. 4).

660

661

Fig. 4 
As shown in Fig. 4, compound $\mathbf{1 0}$ fits snugly in the DAH-type pocket. In this

664

665

666

667

668

669

670

671

672

673

674

675

676

677

678

679

680

681

682

683

684

685

686

687

688

689

690 model, the perfluoropropyl group is surrounded by the hydrophobic region of the LBP. This model is consistent with the results of the QSAR study, which showed that hydrophobic and electrostatic interactions between the substituents at 2-position and the receptor surface are important for activity.

Docking simulation of compound $\mathbf{1 0}$ was also performed against SfEcR, which was constructed from the X-ray crystal structure bound to the IMD-type compound (PDB ID: 3IXP). Although compounds 2 and 9 can dock to the LBP of SfEcR constructed from 3IXP, compound $\mathbf{1 0}$ containing the perfluoropropyl group could not be accommodated in the corresponding pocket. This is likely because of the slightly smaller size of the LBP of 3IXP compared to the DAH binding pocket. Because the protein was treated as a rigid body in the docking simulation using FRED, the initial size of the ligand binding pocket is thought to be critical for docking.

As shown above, compound $\mathbf{2}$ and $\mathbf{9}$ were moderately toxic to S. litura. The dead larva of S. liura treated with compound 2 is shown in Fig. 5. In our previous studies, we synthesized DAH analogs with various substituents at both benzene rings and quantitatively analyzed the structure-activity relationship to identify the essential physicochemical properties for larvicidal activity [33, 34]. QSAR equations showed that when molecular hydrophobicity was high, larvicidal activity against the lepidopteran rice stem borer Chilo suppressalis was also high. Although the optimum hydrophobicity was not derived for the DAH-type compounds, which had limited hydrophobicity (varied A-ring moiety: 2.04-4.68; varied B-ring moiety: 1.99-4.53), there may be an optimum value for the expanded set of compounds with supra-optimum hydrophobicity. In fact, optimum hydrophobicity $\left(\log \mathrm{P}_{\mathrm{opt}}=5.15\right)$ was evaluated for activity against $C$. suppressalis [38]. Although this insect species was different from S. frugiperda used in this study, $\log \mathrm{P}$ values of compounds $\mathbf{4}$ and $\mathbf{5}$ exceeded 5.15, suggesting the presence of optimum hydrophobicity. 
691

692

693

$694\left(\mathrm{pIC}_{50}=7.39\right)$ against Sf-9 cells, but was not toxic to S. litura. This may be because of the 695 facile oxidation of the sulfide moiety to sulfone/sulfoxide through metabolism. We 696

697

698

699

700

701

702

703

704

705

706

707

708

709

710

711

712

713

714

715

716

717

718

Fig. 5 reported that RH-5849 was 10-fold less toxic than tebufenozide against Lepidopteran $C$. suppressalis (Pyralidae; $\mathrm{pLD}_{50}=6.27$ vs. 7.32 ) and 100 -fold less toxic than tebufenozide against S. exigua (Noctuidae; $\mathrm{pLD}_{50}=4.91$ vs. 7.06) [39]. The difference in $\mathrm{pLD}_{50}$ values between RH-5849 and tebufenozide was 100-fold against $S$. litura (Noctuidae), which is consistent with the toxicity results for S. exigua.

\section{Conclusion}

Among 18 synthesized imidazothiadiazole analogs, two compounds with $\mathrm{CF}_{2} \mathrm{CF}_{3}$ and $\mathrm{CF}_{2} \mathrm{CF}_{2} \mathrm{CF}_{3}$ groups showed higher receptor binding activity than ponasterone $\mathrm{A}$ against Lepidoptera Sf-9 cells. The larvicidal activity of the $\mathrm{CF}_{2} \mathrm{CF}_{3}$ analog was determined against $S$. litura larvae in terms of $\mathrm{pLD}_{50}$. It was 5 times more toxic than RH5849, but 20 times less potent than tebufenozide. All compounds, however, did not show strong binding activity against mosquito cells (Diptera) and beetle cells (Coleoptera). This selective toxicity profile among insect orders is similar to that for DAHs. In the structure-activity relationship study, a compound with a primary acrylamide moiety was inactive, but the mono-alkylation of terminal nitrogen of acrylamides (secondary amides) drastically enhanced the activity. Among secondary amides, compounds with isopropyl, $c y c$-butyl and $c y c$-hexyl groups have similar receptor binding activity, but the further alkylation (tertiary amide) was detrimental for the binding. The conversion of amide to thioamide did not have much impact to the activity, but the saturation of olefin moiety and conversion of amide to sulfonamide were also detrimental to the activity. QSAR analysis of the substituent effect at 2-position of the imidazothiadiazole ring indicated that the electron withdrawing and hydrophobic 
719 substituents at this position are favorable for the ligand-receptor binding.

720

\section{Acknowledgement}

722 We are thankful to Drs. Katsuichiro Komatsu and Hideaki Umeyama for supporting the

723 protein modeling using Isolated FAMS. We also thank to Mr. Kiyomitsu Yoshida and Dr.

724 Kazuhisa Kiriyama of Ishihara Sangyo Kaisha, Ltd. for providing eggs of Spodoptera

725 litura. We thank Dr. Keith Wing for the invaluable suggestions. This study was supported

726 in part by the Ministry of Education, Culture, Sports, Science, and Technology of Japan 727 (No. 25450070).

728

729 


\section{References}

1. K.D. Wing, R.A. Slawecki, G.R. Carlson, RH 5849, a Nonsteroidal Ecdysone Agonist: Effects on Larval Lepidoptera, Science 241 (1988) 470-472.

2. A.C.-T. Hsu, 1,2-Diacyl-1-alkylhydrazines, a new class of insect growth regulators. In Synthesis and Chemistry of Agrochemicals II (Baker DR, Fenyes JG \& Moberg WK, eds), American Chemical Society, Washington DC., (1991) pp. 478-490.

3. L. Dinan, Y. Nakagawa, R.E. Hormann, Structure-Activity Relationships of Ecdysteroids and Non-Steroidal Ecdysone Agonists, Adv. Insect Physiol. 43 (2012) 251-298.

4. S.R. Palli, C.M. Tice, V.M. Margam, A.M. Clark, Biochemical mode of action and differential activity of new ecdysone agonists against mosquitoes and moths, Arch. Insect Biochem. Biophys. 58 (2005) 234-242.

5. K. Mikitani, A new nonsteroidal chemical class of ligand for the ecdysteroid receptor 3,5-di-tert-butyl-4-hydroxy- $N$-isobutyl-benzamide shows apparent insect molting hormone activities at molecular and cellular levels, Biochem. Biophys. Res. Commun. 227 (1996) 427-432.

6. C.M. Tice, R.E. Hormann, C.S. Thompson, J.L. Friz, C.K. Cavanaugh, J.A. Saggers, Optimization of $\alpha$-acylaminoketone ecdysone agonists for control of gene expression, Bioorg. Med. Chem. Lett. 13 (2003) 1883-1886.

7. R.E. Hormann, O. Chortyk, D.P. Le, Oxadiazoline ligands for modulating the expression of exogenous genes via an ecdysone receptor complex (2007) US 7,304,162 B2.

8. W. Birru, R.T. Fernley, L.D. Graham, J. Grusovin, R.J. Hill, A. Hofmann, L. Howell, P.J. James, K.E. Jarvis, W.M. Johnson, et al., Synthesis, binding and bioactivity of gamma-methylene gamma-lactam ecdysone receptor ligands: advantages of QSAR models for flexible receptors, Bioorg. Med. Chem. 18 (2010) 5647-5660.

9. G. Holmwood, M. Schindler, Protein structure based rational design of ecdysone agonists, Bioorg. Med. Chem. 17 (2009) 4064-4070.

10. A.K. Gadad, M.B. Palkar, K. Anand, M.N. Noolvi, T.S. Boreddy, J. Wagwade, Synthesis and biological evaluation of 2-trifluoromethyl/sulfonamido-5,6-diaryl 
substituted imidazo[2,1-b]-1,3,4-thiadiazoles: a novel class of cyclooxygenase-2 inhibitors, Bioorg. Med. Chem. 16 (2008) 276-283.

11. M.N. Noolvi, H.M. Patel, N. Singh, A.K. Gadad, S.S. Cameotra, A. Badiger, Synthesis and anticancer evaluation novel 2-cyclopropylimidazo[2,1-b][1,3,4]-thiadiazole derivatives, Eur. J. Med. Chem. 46 (2011) 4411-4418.

12. S.G. Alegaon, K.R. Alagawadi, P.V. Sonkusare, S.M. Chaudhary, D.H. Dadwe, A.S. Shah, Novel imidazo[2,1-b][1,3,4]thiadiazole carrying rhodanine-3-acetic acid as potential antitubercular agents, Bioorg. Med. Chem. Lett. 22 (2012) 1917-1921.

13. T. Fujita, J. Iwasa, C. Hansch, A new substituent constant, $\pi$, derived from partition coefficients, J. Am. Chem. Soc. 86 (1964) 5175-5180.

14. S. Kitamura, T. Harada, H. Hiramatsu, R. Shimizu, H. Miyagawa, Y. Nakagawa, Structural requirement and stereospecificity of tetrahydroquinolines as potent ecdysone agonists, Bioorg. Med. Chem. Lett. 24 (2014) 1715-1718.

15. T. Ogura, Y. Nakagawa, L. Swevers, G. Smagghe, H. Miyagawa, Quantitative evaluation of the molting hormone activity in coleopteran cells established from the Colorado potato beetle, Leptinotarsa decemlineata, Pestic. Biochem. Physiol. 104 (2012) $1-8$.

16. Y. Nakagawa, C. Minakuchi, T. Ueno, Inhibition of $\left[{ }^{3} \mathrm{H}\right]$ ponasterone A binding by ecdysone agonists in the intact Sf-9 cell line, Steroids 65 (2000) 537-542.

17. Y. Nakagawa, C. Minakuchi, K. Takahashi, T. Ueno, Inhibition of [ $\left.{ }^{3} \mathrm{H}\right]$ ponasterone A binding by ecdysone agonists in the intact Kc cell line, Insect Biochem. Mol. Biol. 32 (2002) 175-180.

18. M. Sakuma, Probit analysis of preference data, Appl. Entmol. Zool. 33 (1998) 339-347.

19. M. Asao, R. Shimizu, K. Nakao, T. Fujita, QREG, version 2.05, JCPE Registered Program No. 115, Japan Chemistry Program Exchange," Society for Computer Chemistry, Japan.

20. K. Ogata, H. Umeyama, An automatic homology modeling method consisting of database searches and simulated annealing, J. Mol. Graph. Model. 18 (2000) 258-272.

21. J.H. Chen, P.C. Turner, H.H. Rees, Molecular cloning and induction of nuclear receptors from insect cell lines, Insect Biochem. Mol. Biol. 32 (2002) 657-667.

22. E.J. Pascal, S.A. Valentine, J.A. Brown, A.S. Cockrell, B.D. Johnson, Control of gene 
expression in plants (2002) WO 02/061102 A2.

23. I.M. Billas, T. Iwema, J.M. Garnier, A. Mitschler, N. Rochel, D. Moras, Structural adaptability in the ligand-binding pocket of the ecdysone hormone receptor, Nature 426 (2003) 91-96.

24. C. Browning, E. Martin, C. Loch, J.M. Wurtz, D. Moras, R.H. Stote, A.P. Dejaegere, I.M. Billas, Critical role of desolvation in the binding of 20-hydroxyecdysone to the ecdysone receptor, J. Biol. Chem. 282 (2007) 32924-32934.

25. P.C.D. Hawkins, A.G. Skillman, G. L. Warren, B.A. Ellingson, M.T. Stahl, Conformer generation with OMEGA: algorithm and validation using high quality structures from the protain databank and Cambridge structural database, J. Chem. Inf. Model. 50 (2010) 572-584.

26. M. McGann, FRED pose prediction and virtual screening accuracy, J. Chem. Inf. Model. 51 (2011) 578-596.

27. H.-G. Schwarts, J. Scherkenbeck, G. Holmwood, J. Wieschemeyer, P. Losel, O. Malsam, M.-W. Drewes, K.-H. Kuck, U. Wachendorff-Neumann, C. Arnold, et al. Heterobicyclic acrylamides (2006) WO 2006/094666 A1.

28. W.A. Remers, G.J. Gibs, M.J. Weiss, Preparation of halomethyl-1,3,4-thiadiazoles. Conversion to 2-amino-5-(1-methyl-5-nitro-2-imidazolyl)-1,3,4-thiadiazole, an important antimicrobial agent, J. Het. Chem. 6 (1969)) 835-840.

29. F. Clerici, D. Pocar, M. Guido, A. Loche, V. Perlini, M. Brufani, Synthesis of 2-amino-5-sulfanyl-1,3,4-thiadiazole derivatives and evaluation of their antidepressant and anxiolytic activity, J. Med. Chem. 44 (2001) 931-936.

30. D.C. Reuter, J.E. McIntosh, A.C. Guinn, A.M. Madera, Synthesis of vinyl sulfonamides using the Horner reaction, Synthesis (2003) 2321-2324.

31. M.A. Blanchette, W. Choy, J.T. Davis, A.P. Essenfeld, S. Masamune, W.R. Roush, T. Sakai, Horner-Wadsworth-Emmons Reaction: Use of lithium chloride and an amine for base sensitive compounds, Tetrahedron Lett. 25 (1984) 2183-2186.

32. T. Ogura, Y. Nakagawa, C. Minakuchi, H. Miyagawa, QSAR for binding affinity of substituted dibenzoylhydrazines to intact Sf-9 cells, J. Pestic. Sci. 30 (2005) 1-6.

33. N. Oikawa, Y. Nakagawa, K. Nishimura, T. Ueno, T. Fujita, Quantitative structure-activity studies of insect growth regulators X. Substituent effects on larvicidal activity of 1-tert-butyl-1-(2-chlorobenzoyl)-2-(substituted benzoyl)hydrazines against Chilo suppressalis and design synthesis of potent derivatives, Pestic. Biochem. Physiol. 
48 (1994) 135-144.

34. N. Oikawa, Y. Nakagawa, K. Nishimura, T. UenoT. Fujita, Quantitative structure-activity analysis of larvicidal 1-(substituted benzoyl)-2-benzoyl-1-tert-butylhydrazines against Chilo suppressalis, Pestic. Sci. 41 (1994) 139-148.

35. B. Shimizu, Y. Nakagawa, K. Hattori, K. Nishimura, N. Kurihara, T. Ueno, Molting hormonal and larvicidal activities of aliphatic acyl analogs of dibenzoylhydrazine insecticides, Steroids 62 (1997) 638-642.

36. Y. Nakagawa, Nonsteroidal ecdysone agonists, Vitam. Horm. 73 (2005) 131-173.

37. T. Soin, L. Swevers, G. Kotzia, K. Iatrou, C.R. Janssen, P. Rouge, T. Harada, Y.

NakagawaG. Smagghe, Comparison of the activity of non-steroidal ecdysone agonists between dipteran and lepidopteran insects, using cell-based EcR reporter assays, Pest Manag. Sci. 66 (2010) 1215-1229.

38. Y. Nakagawa, K. Hattori, C. Minakuchi, S. Kugimiya, T. Ueno, Relationships between structure and molting hormonal activity of tebufenozide, methoxyfenozide, and their analogs in cultured integument system of Chilo suppressalis Walker, Steroids 65 (2000) 117-123.

39. G. Smagghe, Y. Nakagawa, B. Carton, A.K. Mourad, T. Fujita, L. Tirry, Comparative ecdysteroid action of ring-substituted dibenzoylhydrazines in Spodoptera exigua, Arch. Insect Biochem. Physiol. 41 (1999) 42-53.

40. T. Ogura, C. Minakuchi, Y. Nakagawa, G. Smagghe, H. Miyagawa, Molecular cloning, expression analysis and functional confirmation of ecdysone receptor and ultraspiracle from the Colorado potato beetle Leptinotarsa decemlineata, FEBS J. 272 (2005) 4114-4128. 


\section{Figure Legends}

Fig. 1. Chemical structures of ecdysone agonists

Fig. 2. Imidazothiadiazole-type compounds synthesized for SAR study

Fig. 3. Summary of structure-activity relationship for the binding activity against Sf-9

Fig. 4. Docking simulation of compound $\mathbf{1 0}$ (colored with green) against SfEcR bound to PonA (left) and DAH (BYI06833; right). Structure colored with gray is PonA and that colored with light brown is BYI06833. Ligand binding domains were modeled from 1R1K (left) and IR20 (right).

Fig. 5. S. litura larva treated with compound $2\left(10^{-5} \mathrm{mmol} / \mathrm{insect}\right)$

Scheme 1. Construction of 2-amino-1,3,4-thiadiazole moiety: (a) $\mathrm{POCl}_{3}$; (b) $\mathrm{CH}_{3} \mathrm{I}, \mathrm{KOH}$, 2-propanol $/ \mathrm{H}_{2} \mathrm{O}$

Scheme 2. Construction of (imidazo[2,1-b][1,3,4]thiadiazol-5-yl)acrylic acid moiety: (a) EtOH; (b) $\mathrm{POCl}_{3}, \mathrm{DMF}$; (c) $\mathrm{CH}_{2}(\mathrm{COOH})_{2}$, piperidine, pyridine

Scheme 3. Synthesis of (imidazo[2,1-b][1,3,4]thiadiazol-5-yl)acrylamides: (a) i. $(\mathrm{COCl})_{2}$, DMF, $\mathrm{CH}_{2} \mathrm{Cl}_{2}$ ii. $\mathrm{NH}_{3}$ aq., $\mathrm{CH}_{2} \mathrm{Cl}_{2}$; (b) amine, EDC, DMAP, $\mathrm{CH}_{2} \mathrm{Cl}_{2}$ 
Scheme 4. Synthesis of sulfoxide 14 and sulfone 15 (a) $m$-CPBA, $\mathrm{CH}_{2} \mathrm{Cl}_{2}$; (b) Oxone ${ }^{\circledR}$, $\mathrm{THF} / \mathrm{MeOH} / \mathrm{H}_{2} \mathrm{O}$

Scheme 5. Synthesis of propionamide 16: (a) $\mathrm{N}_{2} \mathrm{H}_{4}, \mathrm{NaIO}_{4}, \mathrm{MeOH} / \mathrm{H}_{2} \mathrm{O}$ (b) isopropylamine, $\mathrm{EDC}$, DMAP, $\mathrm{CH}_{2} \mathrm{Cl}_{2}$

Scheme 6. Synthesis of thioamide 17: (a) Lawesson's reagent, toluene

Scheme 7. Synthesis of vinyl sulfonamide 18: (a) $\mathrm{Ph}_{2} \mathrm{P}(\mathrm{O}) \mathrm{CH}_{2} \mathrm{SO}_{2} \mathrm{NHBoc}, \mathrm{LiCl}, \mathrm{DBU}$, $\mathrm{CH}_{3} \mathrm{CN}$; (b) $i$-PrOH, DEAD, $\mathrm{PPh}_{3}$, THF; (c) TFA, $\mathrm{CH}_{2} \mathrm{Cl}_{2}$ 
Table 1. Biological activity of synthesized compounds.

\begin{tabular}{|c|c|c|c|c|c|c|c|c|c|}
\hline \multirow[b]{2}{*}{ No } & \multirow[b]{2}{*}{$\mathrm{X}$} & \multirow[b]{2}{*}{$\mathrm{L}$} & \multirow[b]{2}{*}{$\mathrm{R}_{1}$} & \multirow[b]{2}{*}{$\mathrm{R}_{2}$} & \multicolumn{3}{|l|}{$\mathrm{pIC}_{50}(\mathrm{M})$} & \multirow{2}{*}{$\begin{array}{l}\mathrm{pLD}_{50} \\
\text { (mmol/insect) } \\
\text { S. litura }\end{array}$} & \multirow[t]{2}{*}{$\mathrm{Clog} \mathrm{P}$} \\
\hline & & & & & Sf-9 & NIAS-AeA12 & BCIRL-Lepd-SL1 & & \\
\hline 1 & $\mathrm{CF}_{3}$ & trans $-\mathrm{CH}=\mathrm{CH}-\mathrm{C}(=\mathrm{O})-$ & $\mathrm{H}$ & $\mathrm{H}$ & $<4.9(12 \%)$ & n.d. ${ }^{\mathrm{a}}$ & n.d. & n.d. & 3.56 \\
\hline 2 & $\mathrm{CF}_{3}$ & trans $-\mathrm{CH}=\mathrm{CH}-\mathrm{C}(=\mathrm{O})-$ & $\mathrm{H}$ & $i-\operatorname{Pr}$ & $8.03^{\mathrm{d}}$ & 7.10 & 4.88 & 5.16 & 4.63 \\
\hline 3 & $\mathrm{CF}_{3}$ & trans $-\mathrm{CH}=\mathrm{CH}-\mathrm{C}(=\mathrm{O})-$ & $\mathrm{H}$ & $c-\mathrm{Bu}$ & 7.71 & 6.74 & $\approx 4.08(49 \%)$ & $<4.00(45 \%)$ & 4.70 \\
\hline 4 & $\mathrm{CF}_{3}$ & trans $-\mathrm{CH}=\mathrm{CH}-\mathrm{C}(=\mathrm{O})-$ & $\mathrm{H}$ & $c$-Hex & 7.65 & 6.05 & $<4.60(27 \%)$ & $<4.30(10 \%)$ & 5.82 \\
\hline 5 & $\mathrm{CF}_{3}$ & trans $-\mathrm{CH}=\mathrm{CH}-\mathrm{C}(=\mathrm{O})-$ & $\mathrm{H}$ & $\mathrm{Ph}$ & 7.08 & 5.94 & $<4.38(10 \%)$ & $<4.30(0 \%)$ & 5.65 \\
\hline 6 & $\mathrm{CF}_{3}$ & trans $-\mathrm{CH}=\mathrm{CH}-\mathrm{C}(=\mathrm{O})-$ & $\mathrm{CH}_{3}$ & $i-\operatorname{Pr}$ & 5.20 & $\approx 4.38(51 \%)$ & $<4.38(39 \%)$ & $<4.30(25 \%)$ & 4.52 \\
\hline 7 & $\mathrm{CF}_{3}$ & trans $-\mathrm{CH}=\mathrm{CH}-\mathrm{C}(=\mathrm{O})-$ & $-\left(\mathrm{CH}_{2}\right)_{5}^{-}$ & & 5.24 & $\approx 4.08(53 \%)$ & $\approx 4.08(55 \%)$ & $<4.48(0 \%)$ & 4.88 \\
\hline 8 & $\mathrm{CF}_{3}$ & trans $-\mathrm{CH}=\mathrm{CH}-\mathrm{C}(=\mathrm{O})-$ & $-\left(\mathrm{CH}_{2}\right)_{2}-\mathrm{O}$ & $-\left(\mathrm{CH}_{2}\right)_{2}^{-}$ & 5.02 & $<4.08(24 \%)$ & $<4.08(17 \%)$ & $<4.48(10 \%)$ & 3.75 \\
\hline 9 & $\mathrm{CF}_{2} \mathrm{CF}_{3}$ & trans $-\mathrm{CH}=\mathrm{CH}-\mathrm{C}(=\mathrm{O})-$ & $\mathrm{H}$ & $i-\operatorname{Pr}$ & $8.35^{\mathrm{e}}$ & 7.52 & 4.72 & 5.03 & 5.00 \\
\hline
\end{tabular}




\begin{tabular}{|c|c|c|c|c|c|c|c|c|c|}
\hline 10 & $\mathrm{CF}_{2} \mathrm{CF}_{2} \mathrm{CF}_{3}$ & trans $-\mathrm{CH}=\mathrm{CH}-\mathrm{C}(=\mathrm{O})-$ & $\mathrm{H}$ & $i-\operatorname{Pr}$ & 8.48 & n.d. & n.d. & n.d. & 5.23 \\
\hline 11 & $\mathrm{H}$ & trans $-\mathrm{CH}=\mathrm{CH}-\mathrm{C}(=\mathrm{O})-$ & $\mathrm{H}$ & $i-\operatorname{Pr}$ & 4.79 & n.d. & $<4.60(4.6 \%)$ & n.d. & 3.74 \\
\hline 12 & $\mathrm{CH}_{3}$ & trans $-\mathrm{CH}=\mathrm{CH}-\mathrm{C}(=\mathrm{O})-$ & $\mathrm{H}$ & $i-\operatorname{Pr}$ & 5.44 & $<4.38(42 \%)$ & $\approx 4.38(47 \%)$ & $<4.30(0 \%)$ & 4.24 \\
\hline 13 & $\mathrm{SCH}_{3}$ & trans $-\mathrm{CH}=\mathrm{CH}-\mathrm{C}(=\mathrm{O})-$ & $\mathrm{H}$ & $i-\operatorname{Pr}$ & $7.39^{\mathrm{f}}$ & 6.39 & $<4.90(35 \%)$ & $<4.30(0 \%)$ & 4.44 \\
\hline 14 & $\mathrm{~S}(=\mathrm{O}) \mathrm{CH}_{3}$ & trans $-\mathrm{CH}=\mathrm{CH}-\mathrm{C}(=\mathrm{O})-$ & $\mathrm{H}$ & $i-\operatorname{Pr}$ & 4.79 & $<4.60(38 \%)$ & $<4.90(16 \%)$ & $<4.00(0 \%)$ & 2.44 \\
\hline 15 & $\mathrm{SO}_{2} \mathrm{CH}_{3}$ & trans $-\mathrm{CH}=\mathrm{CH}-\mathrm{C}(=\mathrm{O})-$ & $\mathrm{H}$ & $i-\operatorname{Pr}$ & 4.76 & $>4.38(97 \%)$ & $<4.90(11 \%)$ & $<4.30(0 \%)$ & 2.26 \\
\hline 16 & $\mathrm{CF}_{3}$ & $-\mathrm{CH}_{2}-\mathrm{CH}_{2}-\mathrm{C}(=\mathrm{O})-$ & $\mathrm{H}$ & $i-\operatorname{Pr}$ & 5.83 & $\approx 4.08(56 \%)$ & $<4.08(25 \%)$ & $<4.48(5 \%)$ & 3.54 \\
\hline 17 & $\mathrm{CF}_{3}$ & trans $-\mathrm{CH}=\mathrm{CH}-\mathrm{C}(=\mathrm{S})-$ & $\mathrm{H}$ & $i-\operatorname{Pr}$ & 7.51 & $>4.60(99 \%)$ & $<4.90(38 \%)$ & n.d. & 4.85 \\
\hline 18 & $\mathrm{CF}_{3}$ & trans $-\mathrm{CH}=\mathrm{CH}-\mathrm{SO}_{2}-$ & $\mathrm{H}$ & $i-\operatorname{Pr}$ & 4.98 & n.d. & n.d. & n.d. & 4.23 \\
\hline 19 & Ponasterone A & & & & 8.05 & $9.01^{\mathrm{b}}$ & $8.13^{\mathrm{c}}$ & n.d. & 0.49 \\
\hline 20 & 20-Hydroxyec & dysone & & & 6.78 & n.d. & $6.36^{\mathrm{c}}$ & n.d. & -1.72 \\
\hline 21 & Tebufenozide & & & & 8.81 & 7.12 & 5.18 & $6.47^{\mathrm{g}}$ & 4.51 \\
\hline 22 & RH-5849 & & & & 6.44 & n.d. & n.d. & $4.41^{\mathrm{g}}$ & 2.45 \\
\hline
\end{tabular}

${ }^{a}$ Not determined

${ }^{\mathrm{b}}$ From Ref. [11]

${ }^{\mathrm{c}}$ Against the in vitro translated EcR/USP heterodimers. From Ref. [40]

${ }^{\mathrm{d}}$ Mean of 7.78, 8.04 and 8.27 
${ }^{\mathrm{e}}$ Mean of 8.32 and 8.38

${ }^{\mathrm{f}}$ Mean of 7.37 and 7.40

${ }^{\mathrm{g}}$ Dose-response relationship was derived using ten larvae for each dose. 
Table 2. Physicochemical parameters for QSAR calculation and prediction of the binding activity by Eq. 2.

\begin{tabular}{|c|c|c|c|c|c|c|}
\hline \multicolumn{2}{|c|}{ Compounds } & \multicolumn{2}{|c|}{ Physicochemical parameter } & \multicolumn{3}{|c|}{ Binding affinity $\left(\mathrm{pIC}_{50}\right)$} \\
\hline No & Substituents & $\Delta \mathrm{Clog} \mathrm{P}$ & $\mathrm{F}$ & Obsd & Calcd (Eq.2) & $\Delta^{\mathrm{a}}$ \\
\hline 11 & $\mathrm{H}$ & 0.00 & 0.00 & 4.79 & 4.87 & -0.08 \\
\hline 12 & $\mathrm{CH}_{3}$ & 0.50 & 0.01 & 5.44 & 5.67 & -0.23 \\
\hline 13 & $\mathrm{SCH}_{3}$ & 0.70 & 0.23 & 7.39 & 6.84 & 0.55 \\
\hline 14 & $\mathrm{SOCH}_{3}$ & -1.30 & 0.52 & 4.79 & 4.94 & -0.15 \\
\hline 15 & $\mathrm{SO}_{2} \mathrm{CH}_{3}$ & -1.48 & 0.53 & 4.76 & 4.70 & -0.06 \\
\hline 2 & $\mathrm{CF}_{3}$ & 0.89 & 0.38 & 8.03 & 7.72 & 0.31 \\
\hline 9 & $\mathrm{CF}_{2} \mathrm{CF}_{3}$ & 1.26 & 0.44 & 8.35 & 8.51 & -0.16 \\
\hline 10 & $\mathrm{CF}_{2} \mathrm{CF}_{2} \mathrm{CF}_{3}$ & 1.49 & 0.42 & 8.48 & 8.78 & -0.30 \\
\hline
\end{tabular}

${ }^{\mathrm{a}}$ The difference between observed and calculated value $\left[\mathrm{pIC}_{50}(\mathrm{obsd})-\mathrm{pIC}_{50}(\right.$ calcd by Eq. 2$\left.)\right]$. 
<smiles>[R]C(C)(C)CCC[C@H](O)[C@@](C)(O)[C@H]1CC[C@]2(O)C3=CC(=O)[C@H]4C[C@@H](O)[C@@H](O)C[C@]4(C)[C@H]3CC[C@]12C</smiles>

20-Hydroxyecdysone $(\mathrm{R}=\mathrm{OH})$ Ponasterone A $(\mathrm{R}=\mathrm{H})$

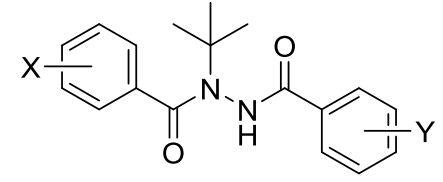

Diacylhydrazine (DAH)

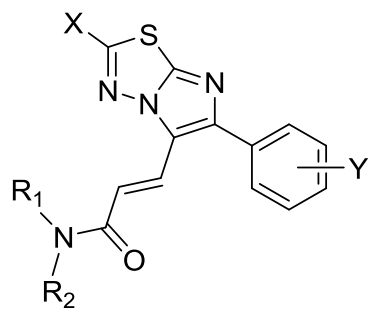

Imidazothiadiazole (ITD)

Fig. 1. Chemical structures of ecdysone agonists<smiles>[X]c1nn2c(/C=C/C(=O)N([R])[R])c(-c3ccccc3Cl)nc2s1</smiles><smiles>CC(C)NC(=O)CCc1c(-c2ccccc2Cl)nc2sc(C(F)(F)F)nn12</smiles><smiles>CC(C)NC(=S)/C=C/c1c(-c2ccccc2Cl)nc2sc(C(F)(F)F)nn12</smiles><smiles>CC(C)NS(=O)(=O)/C=C/c1c(-c2ccccc2Cl)nc2sc(C(F)(F)F)nn12</smiles>

Fig. 2. Imidazothiadiazole-type compounds synthesized for SAR study 


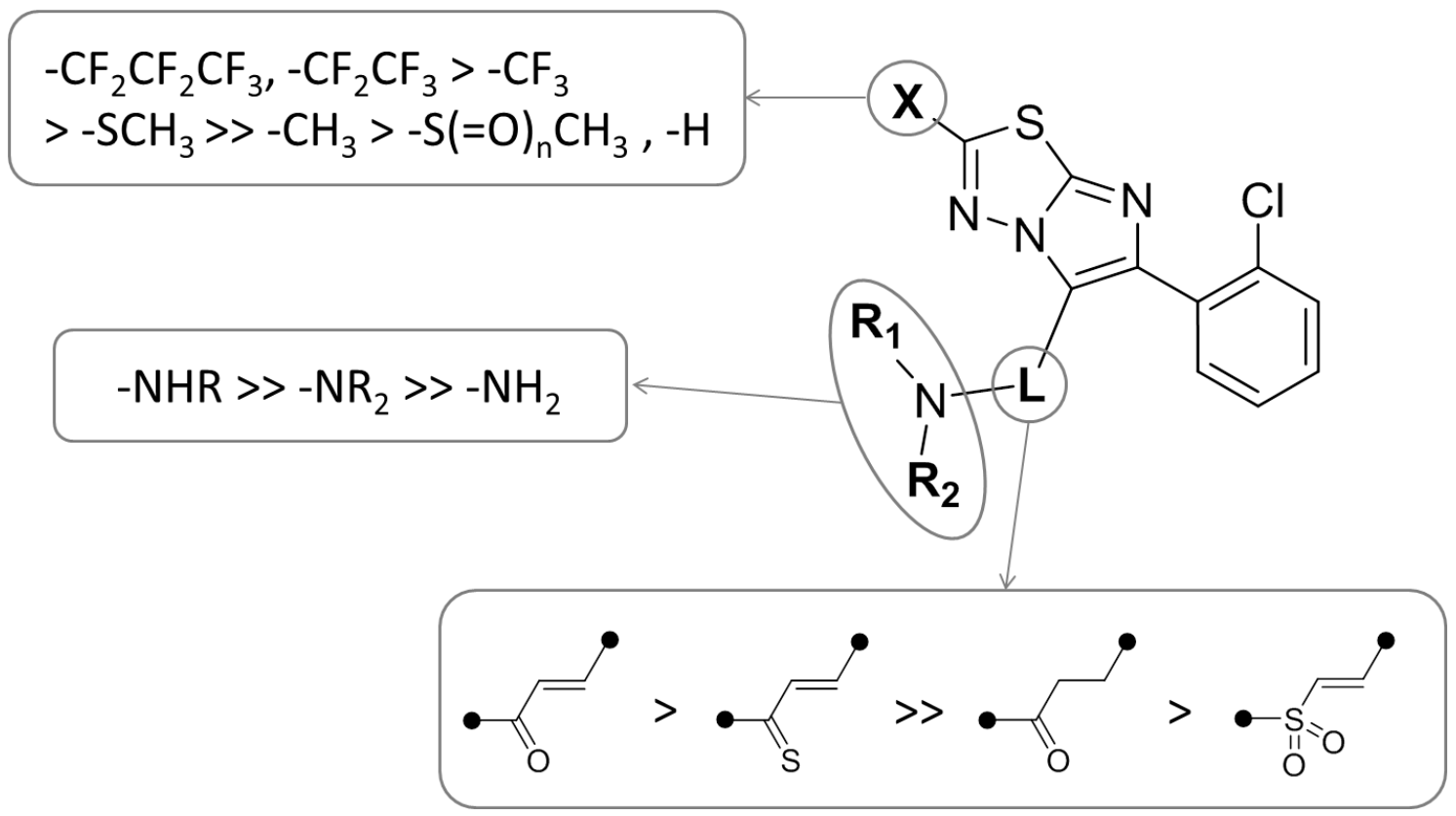

Fig. 3. Summary of structure-activity relationship for the binding activity against Sf-9
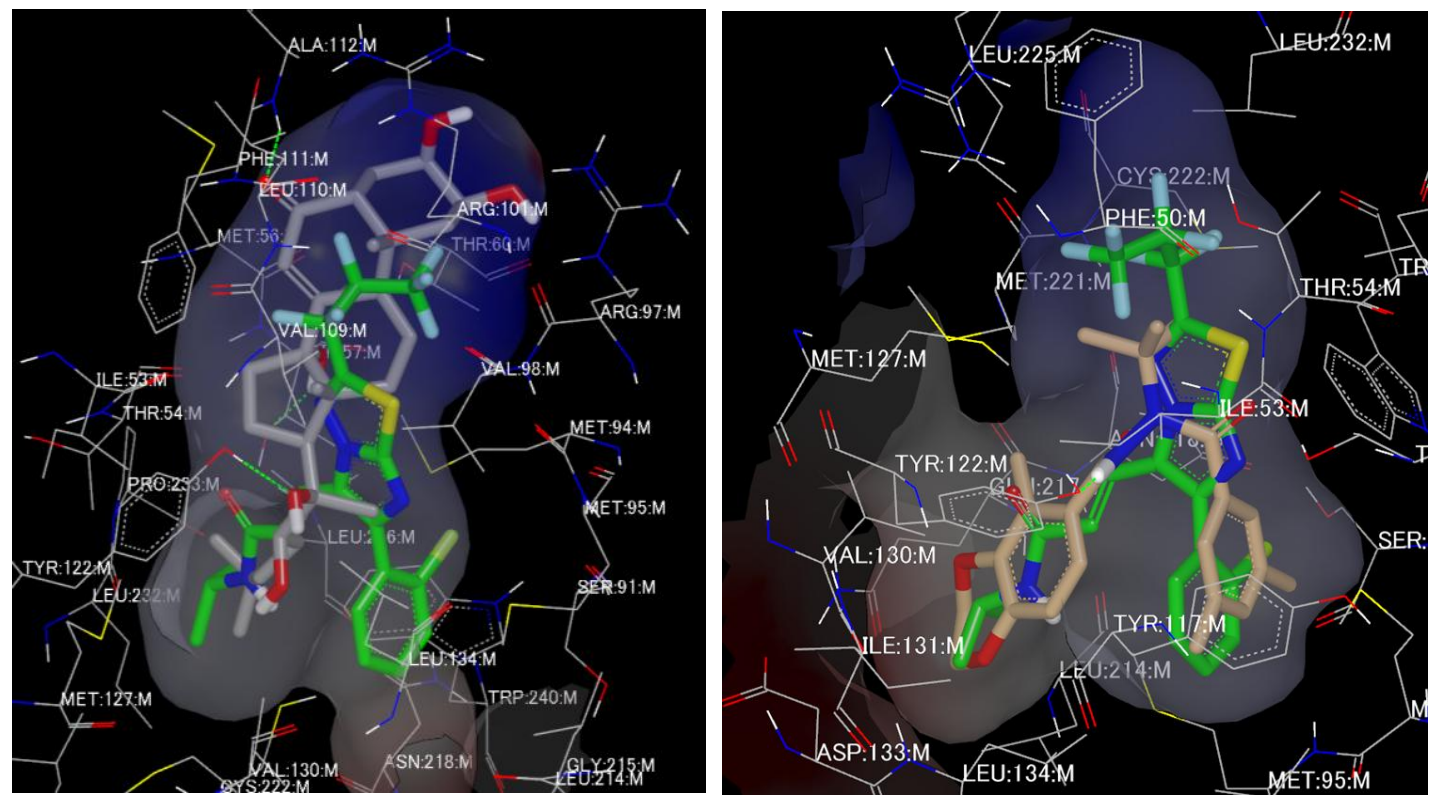

Fig. 4. Docking simulation of compound $\mathbf{1 0}$ (colored with green) against SfEcR bound to PonA (left) and DAH (BYI06833; right). Structure colored with gray is PonA and that colored with light brown is BYI06833. Ligand binding domains were modeled from 1R1K (left) and IR20 (right). 


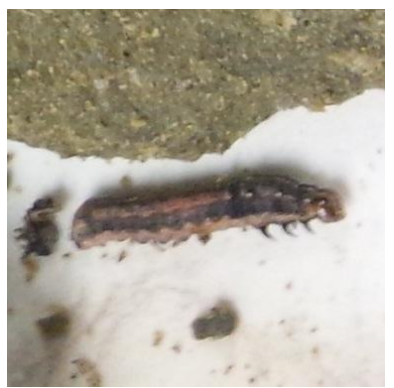

Fig. 5 S. litura Larva treated with compound $2\left(10^{-5} \mathrm{mmol} / \mathrm{insect}\right)$

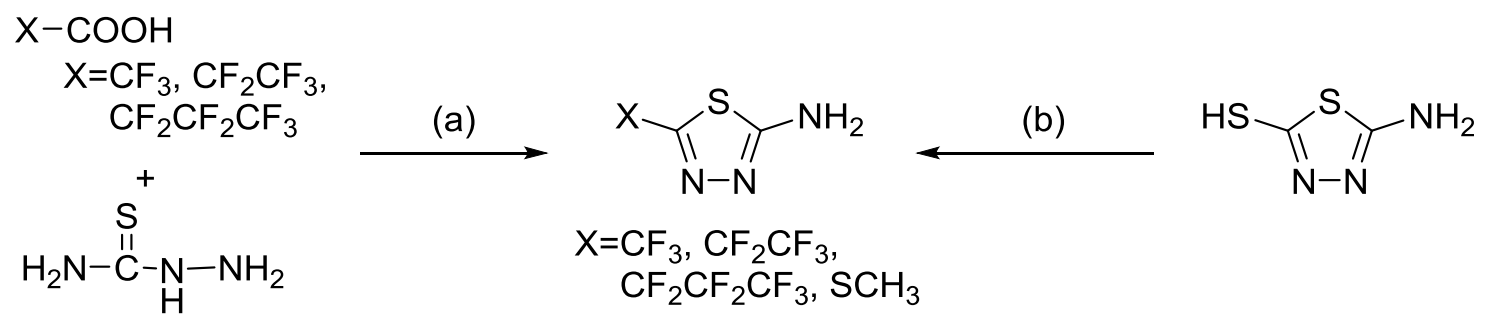

Scheme 1. Construction of 2-amino-1,3,4-thiadiazole moiety: (a) $\mathrm{POCl}_{3}$; (b) $\mathrm{CH}_{3} \mathrm{I}, \mathrm{KOH}$, 2-propanol $/ \mathrm{H}_{2} \mathrm{O}$

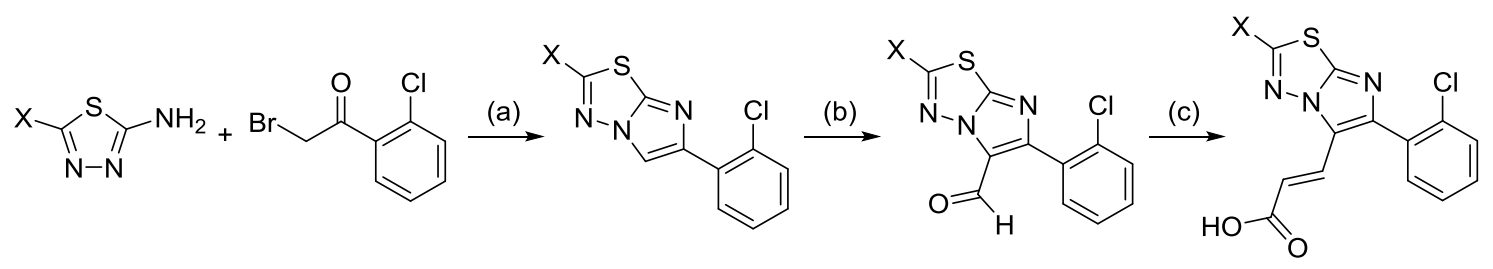

Scheme 2. Construction of (imidazo[2,1-b][1,3,4]thiadiazol-5-yl)acrylic acid moiety: (a) EtOH; (b) $\mathrm{POCl}_{3}, \mathrm{DMF}$; (c) $\mathrm{CH}_{2}(\mathrm{COOH})_{2}$, piperidine, pyridine 
<smiles>[X]c1nn2c(/C=C/C(=O)O)c(-c3ccccc3Cl)nc2s1</smiles><smiles>C[13CH][13CH]</smiles><smiles>[X]c1nn2c(/C=C/C(=O)N([R])[R])c(-c3ccccc3Cl)nc2s1</smiles>

Scheme 3. Synthesis of (imidazo[2,1-b][1,3,4]thiadiazol-5-yl)acrylamides: (a) i. $(\mathrm{COCl})_{2}$, DMF, $\mathrm{CH}_{2} \mathrm{Cl}_{2}$ ii. $\mathrm{NH}_{3}$ aq., $\mathrm{CH}_{2} \mathrm{Cl}_{2}$; (b) amine, EDC, DMAP, $\mathrm{CH}_{2} \mathrm{Cl}_{2}$
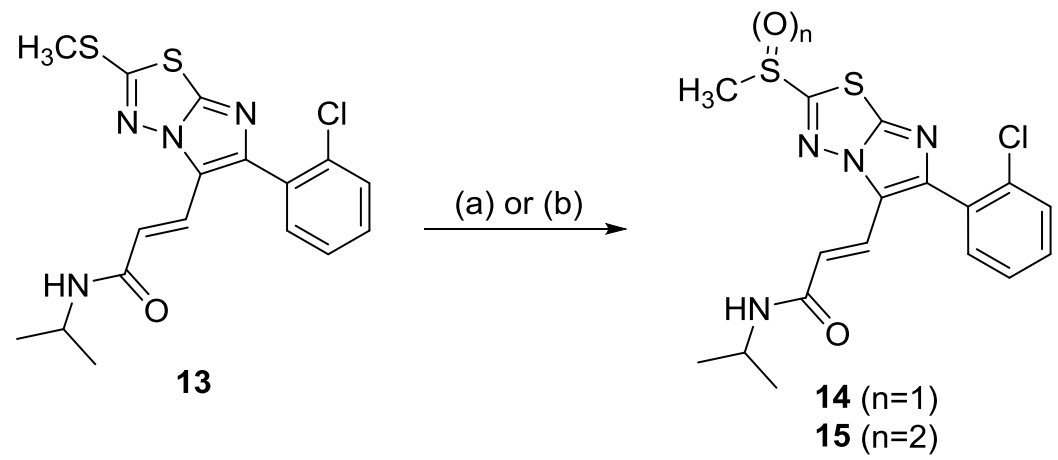

Scheme 4. Synthesis of sulfoxide 14 and sulfone 15: (a) $m$-CPBA, $\mathrm{CH}_{2} \mathrm{Cl}_{2}$; (b) Oxone ${ }^{\circledR}$, $\mathrm{THF} / \mathrm{MeOH} / \mathrm{H}_{2} \mathrm{O}$<smiles>O=C(O)/C=C/c1c(-c2ccccc2Cl)nc2sc(C(F)(F)F)nn12</smiles><smiles>CC(C)NC(=O)CCc1c(-c2ccccc2Cl)nc2sc(C(F)(F)F)nn12</smiles>

Scheme 5. Synthesis of propionamide 16: (a) $\mathrm{N}_{2} \mathrm{H}_{4}, \mathrm{NaIO}_{4}, \mathrm{MeOH} / \mathrm{H}_{2} \mathrm{O}$ (b) isopropylamine, $\mathrm{EDC}$, DMAP, $\mathrm{CH}_{2} \mathrm{Cl}_{2}$ 

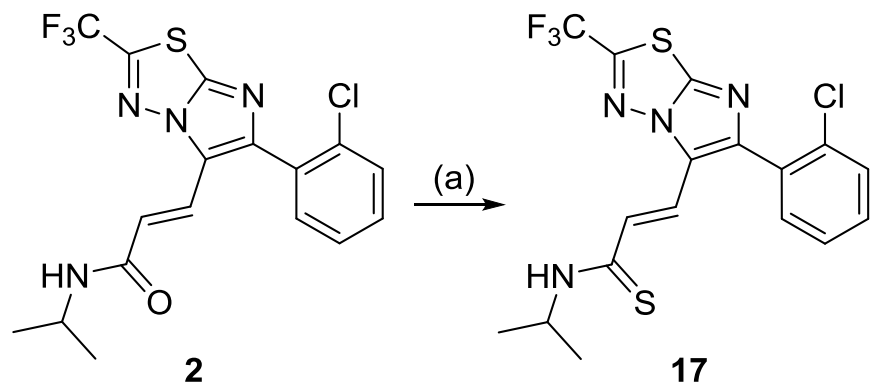

Scheme 6. Synthesis of thioamide 17: (a) Lawesson's reagent, toluene<smiles>Cc1cccc(-c2nc3sc(C(F)(F)F)nn3c2C=O)c1Cl</smiles><smiles>CC(C)(C)OC(=O)NS(=O)(=O)/C=C/c1c(-c2cccc(Cl)c2Cl)nc2sc(C(F)(F)F)nn12</smiles><smiles>CC(C)NS(=O)(=O)/C=C/c1c(-c2ccccc2Cl)nc2sc(C(F)(F)F)nn12</smiles>

Scheme 7. Synthesis of vinyl sulfonamide 18: (a) $\mathrm{Ph}_{2} \mathrm{P}(\mathrm{O}) \mathrm{CH}_{2} \mathrm{SO}_{2} \mathrm{NHBoc}, \mathrm{LiCl}, \mathrm{DBU}$, $\mathrm{CH}_{3} \mathrm{CN}$; (b) $i$-PrOH, DEAD, $\mathrm{PPh}_{3}$, THF; (c) TFA, $\mathrm{CH}_{2} \mathrm{Cl}_{2}$ 